\title{
A Hamiltonian-preserving scheme for high frequency elastic waves in heterogeneous media*
}

\author{
Shi Jin and Xiaomei Liao ${ }^{\dagger}$
}

February 27, 2006

\begin{abstract}
We develop a class of Hamiltonian-preserving numerical schemes for high frequency elastic waves in heterogeneous media. The approach is based on the high frequency approximation governed by the Liouville equations with singular coefficients due to material interfaces. As previously done by Jin and Wen [10, 12], we build into the numerical flux the wave scattering information at the interface, and use the Hamiltonian preserving principle to couple the wave numbers at both sides of the interface. This gives a class of numerical schemes that allows a hyperbolic CFL condition, is positive and $l^{\infty}$ stable, and captures correctly wave scattering at the interface with a sharp numerical resolution. We also extend the method to curved interfaces. Numerical experiments are carried out to study the numerical convergence and accuracy.
\end{abstract}

\section{Introduction}

The propagation of waves in elastic solids plays an important role in seismology. In this paper, we are interested in the high frequency elastic waves in two space dimension, which, in the high frequency limit, are governed by a system of Liouville equations [22]:

$$
\begin{aligned}
& \frac{\partial a^{p}}{\partial t}+\nabla_{\mathbf{k}} H_{p} \cdot \nabla_{\mathbf{x}} a^{p}-\nabla_{\mathbf{x}} H_{p} \cdot \nabla_{\mathbf{k}} a^{p}=0 \\
& \frac{\partial a^{s}}{\partial t}+\nabla_{\mathbf{k}} H_{s} \cdot \nabla_{\mathbf{x}} a^{s}-\nabla_{\mathbf{x}} H_{s} \cdot \nabla_{\mathbf{k}} a^{s}=0,
\end{aligned}
$$

where $\mathbf{x}, \mathbf{k} \in \mathbf{R}^{2}, a^{p}, a^{s}$ are the phase space energy densities for $\mathrm{P}$-wave and $\mathrm{S}$-wave respectively and the Hamiltonians $H_{p}$ and $H_{s}$ possess the form

$$
H_{p}(\mathbf{x}, \mathbf{k})=c^{p}(\mathbf{x})|\mathbf{k}|, \quad H_{s}(\mathbf{x}, \mathbf{k})=c^{s}(\mathbf{x})|\mathbf{k}|
$$

\footnotetext{
*Research supported in part by NSF grant No. DMS-0305080.

$\dagger$ Department of Mathematics, University of Wisconsin, Madison, WI 53706, USA. Email: jin@math.wisc.edu, liao@math.wisc.edu.
} 
with

$$
c^{p}(\mathbf{x})=\sqrt{(\lambda(\mathbf{x})+2 \mu(\mathbf{x})) / \rho(\mathbf{x})} \text { and } \quad c^{s}(\mathbf{x})=\sqrt{\mu(\mathbf{x}) / \rho(\mathbf{x})}
$$

being the local wave speeds respectively for longitudinal wave (P-wave) and transverse shear wave (S-wave). Here $\lambda(\mathbf{x})$ and $\mu(\mathbf{x})$ are the Lamé parameters and $\rho(\mathbf{x})$ is the density. We are concerned with the case when $c^{p}(\mathbf{x})$ and $c^{s}(\mathbf{x})$ contain discontinuities but have bounded variations due to different media. This discontinuity will generate an interface, and as is well known, $\mathrm{P}$ and $\mathrm{S}$ wave modes interact at this interface, namely, a $\mathrm{P}$-wave with wave number $\mathbf{k}$ can scatter into a $\mathrm{S}$-wave with wave number $\mathbf{p}$ with the same Hamiltonian, i.e.

$$
c^{p}(\mathbf{x})|\mathbf{k}|=c^{s}(\mathbf{x})|\mathbf{p}|
$$

and vice versa.

Following [10] and [12], we construct a numerical scheme which is effective to deal with the mode conversion between $\mathrm{P}$-wave and $\mathrm{S}$-wave at the interface, with computable energy partition coefficients [2]. The idea is to build the wave behavior at the interface, which involves the scattering matrix, into the numerical flux, and use the Hamiltonian preserving condition (1.3) to determine wave numbers on one side of the interface from the other side. The scheme, similar to that in [12], allows a typical hyperbolic stability condition $\Delta t=O(|\Delta \mathbf{x}|,|\Delta \mathbf{k}|)$ with $\Delta \mathbf{x}=(\Delta x, \Delta y)$ the mesh size in physical space (likewise for the definition of $\Delta \mathbf{k}$ ) and $\Delta t$ the time step, under which the positivity and $l^{\infty}$ stability are also established.

We will start with the full physical model of elastic waves in $3 \mathrm{D}$ and show how it can be reduced to the two dimensional plane-strain model. This is a reasonable model for plane waves propagating through a three-dimensional elastic body in cases where there is no variation in the $z$-direction, for example, if the $x$ - $y$ plane is a representative slice through a three-dimensional solid with essentially infinite extent in the $z$-direction, which may occur in modelling large scale seismic waves in the earth.

There are a lot of well developed theories for elastic waves in both homogeneous and heterogeneous media, see [1], [2], [4], [7], [14]. Many numerical methods have been introduced to handle effectively wave propagations through heterogeneous media, including acoustic waves and elastic waves, see the recent works [16, 18, 25] and references therein. Our approach bears some similarity with the immersed interface method [17], in that the interface condition is built into the numerical flux. Unlike these earlier works, which were most effective for low frequency waves, our approach is advantageous for high frequency elastic waves. Using the high frequency limit, the Liouville equations, our numerical methods do not need to resolve the short wave length.

For analytic study of high frequency limits of waves through interfaces or boundaries, with transmissions and reflections, we refer to [3, 19, 23].

This paper is organized as follows. In Section 2, we introduce the physical model of elastic wave in 3D and 2D, and its behavior at an interface. The high frequency approximation by the Wigner transform and by the WKB expansion are presented in Section 3. In Section 4, we illustrate the principle to design our numerical scheme and the detailed algorithm, and prove its positivity and $l^{\infty}$ stability. This method is 
extended to curved interfaces in Section 5. Numerical examples are given in Section 6 to verify the convergence and accuracy of the scheme. We conclude in Section 7.

\section{Elastic waves in physical space and their behavior at an interface}

\subsection{Elastic waves in physical space}

The 3D equations of motion for small displacements $\mathbf{u}(t, x)=\left(u_{1}, u_{2}, u_{3}\right)$ of an isotropic elastic medium are given by

$$
\rho \frac{\partial^{2} u_{i}}{\partial t^{2}}=\frac{\partial}{\partial x_{i}}(\lambda \operatorname{div} \mathbf{u})+\sum_{j} \frac{\partial}{\partial x_{j}}\left(\mu\left(\frac{\partial u_{j}}{\partial x_{i}}+\frac{\partial u_{i}}{\partial x_{j}}\right)\right), \quad i=1,2,3 .
$$

where $\rho(\mathbf{x})$ is the density, $\lambda(\mathbf{x})$ and $\mu(\mathbf{x})$ are the Lamé parameters of the medium.

In the homogeneous medium, $\lambda$ and $\mu$ are constants independent of $\mathbf{x}$. So we can write (2.1) in a vector form:

$$
\rho \frac{\partial^{2} \mathbf{u}}{\partial t^{2}}=(\lambda+\mu) \nabla(\nabla \cdot \mathbf{u})+\mu \nabla^{2} \mathbf{u}
$$

For plane waves, the function

$$
\mathbf{u}(\mathbf{x}, t)=f(\mathbf{x} \cdot \mathbf{p}-c t) \mathbf{d}
$$

represents a wave propagating with phase velocity $c$ in the direction of the unit vector $\mathbf{p}$, while $\mathbf{d}$ is a unit vector that defines the direction of the displacement.

Substituting (2.3) into (2.2) and obtaining :

$$
\left[(\lambda+\mu)(\mathbf{p} \cdot \mathbf{d}) \mathbf{p}+\mu \mathbf{d}-\rho c^{2} \mathbf{d}\right] f^{\prime \prime}(\mathbf{x} \cdot \mathbf{p}-c t)=0
$$

Hence

$$
(\lambda+\mu)(\mathbf{p} \cdot \mathbf{d}) \mathbf{p}+\left(\mu-\rho c^{2}\right) \mathbf{d}=0 .
$$

Since $\mathbf{p}$ and $\mathbf{d}$ are different unit vectors, Eq.(2.5) can be satisfied in two ways only. One way is to set

$$
\mathbf{d}= \pm \mathbf{p}
$$

Eq.(2.5) then yields

$$
c^{2}=\left(c^{p}\right)^{2}=(\lambda+2 \mu) / \rho .
$$

In this case the motion is parallel to the direction of propagation, and the wave is therefore called a longitudinal or L wave (primary wave, i.e. P-wave in some context). If $\mathbf{d} \neq \pm \mathbf{p}$, both terms in (2.5) have to vanish, yielding

$$
\mathbf{p} \cdot \mathbf{d}=0 \quad \text { and } \quad c^{2}=\left(c^{s}\right)^{2}=\mu / \rho .
$$


Since now the motion is normal to the direction of propagation, the wave is called a transverse or T-wave (secondary wave i.e. S-wave in some context). Usually we choose the $x y$-plane to contain the vector $\mathbf{p}$, and consider transverse motions in the $x y$-plane or normal to the $x y$-plane. It is called "vertically" polarized transverse or TV(SV) wave if the wave motion in the $x y$-plane is transverse, while a wave motion normal to the $x y$-plane is called a "horizontally" polarized transverse or $\mathrm{TH}(\mathrm{SH})$ wave.

Hence if only in-plane motions are considered, one can reduce the full 3-D model into the model in two dimensional plane by setting $\partial / \partial x_{3} \equiv 0$ and $u_{3} \equiv 0$ in Eq.(2.1) to get:

$$
\left\{\begin{array}{l}
\rho \frac{\partial^{2} u_{1}}{\partial t^{2}}=\frac{\partial}{\partial x_{1}}\left(\lambda\left(\frac{\partial u_{1}}{\partial x_{1}}+\frac{\partial u_{2}}{\partial x_{2}}\right)\right)+\sum_{j=1}^{2} \frac{\partial}{\partial x_{j}}\left(\mu\left(\frac{\partial u_{j}}{\partial x_{1}}+\frac{\partial u_{1}}{\partial x_{j}}\right)\right), \\
\rho \frac{\partial^{2} u_{2}}{\partial t^{2}}=\frac{\partial}{\partial x_{2}}\left(\lambda\left(\frac{\partial u_{1}}{\partial x_{1}}+\frac{\partial u_{2}}{\partial x_{2}}\right)\right)+\sum_{j=1}^{2} \frac{\partial}{\partial x_{j}}\left(\mu\left(\frac{\partial u_{j}}{\partial x_{2}}+\frac{\partial u_{2}}{\partial x_{j}}\right)\right) .
\end{array}\right.
$$

This system, incorporating both $\mathrm{P}$-wave and SV-wave in plane, is particularly named plane strain model, which is the focus of our study in this paper.

\subsection{Behavior of elastic waves at an interface}

It is well known that when an incident longitudinal wave (P-wave) hits an interface of two solids, it gives rise to the reflection and transmission of both a longitudinal and a transverse wave. This wave-splitting effect happens also for an incident transverse wave (S-wave). Here we concentrate on the reflection and transmission of P-SV wave across a solid-solid interface in the plane. The complete system of incident and scattered plane $\mathrm{P}-\mathrm{SV}$ waves is shown in Figure 1. The associated scattering matrix is

$$
\left(\begin{array}{llll}
\grave{P} \dot{P} & \grave{S} \dot{P} & \dot{P} P & \dot{S} P \\
\grave{P} \dot{S} & \grave{S} \dot{S} & \dot{P} \dot{S} & \dot{S} \dot{S} \\
\grave{P} \grave{P} & \grave{S} \grave{P} & \dot{P} \grave{P} & \dot{S} \grave{P} \\
\grave{P} \grave{S} & \grave{S} \grave{S} & \dot{P} \grave{S} & \dot{S} \grave{S}
\end{array}\right)
$$

in which each entry means the ratio of displacement amplitude when wave splits, which can be obtained from the continuity of displacement and traction. Each column of the scattering matrix represents the four waves scattered away from the interface by a particular type of incident wave. The details in manipulation of these 16 coefficients can be found in [2]. Below we present the expressions for the first two columns of (2.9) which will be used later in our numerical scheme: 


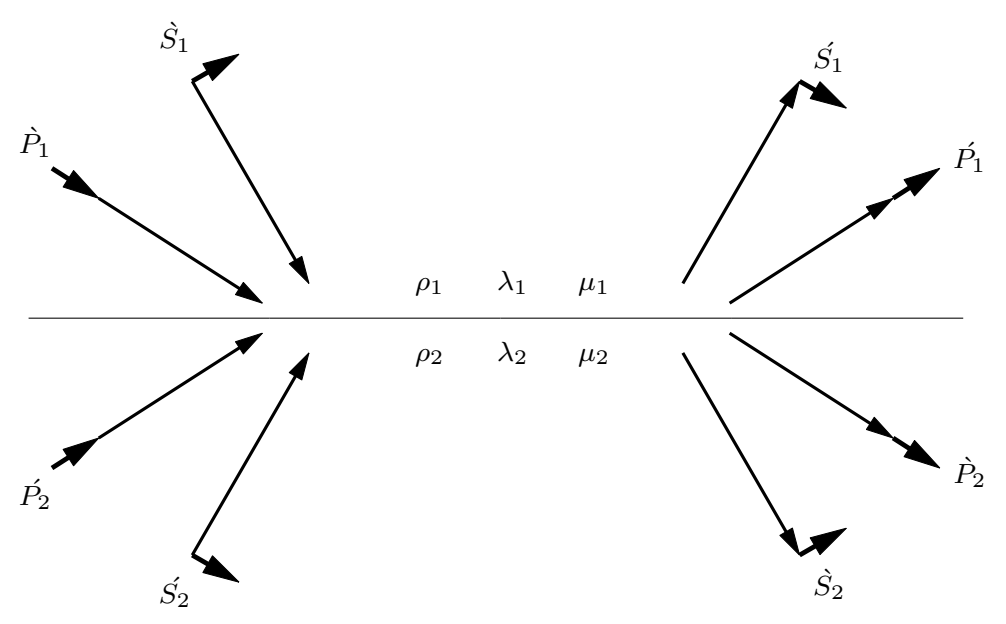

Figure 1: The complete system of incident and scattered plane P-SV waves hitting the interface. Short arrows show the direction of particle motion; long arrows show the direction of propagation.

$$
\begin{aligned}
& \grave{P} \dot{P}=\left[\left(b \frac{\cos i_{1}}{\alpha_{1}}-c \frac{\cos i_{2}}{\alpha_{2}}\right) F-\left(a+d \frac{\cos i_{1}}{\alpha_{1}} \frac{\cos j_{2}}{\beta_{2}}\right) H p^{2}\right] / D \\
& \grave{P} \dot{S}=-2 \frac{\cos i_{1}}{\alpha_{1}}\left(a b+c d \frac{\cos i_{2}}{\alpha_{2}} \frac{\cos j_{2}}{\beta_{2}}\right) p \alpha_{1} /\left(\beta_{1} D\right), \\
& \grave{P} \grave{P}=2 \rho_{1} \frac{\cos i_{1}}{\alpha_{1}} F \alpha_{1} /\left(\alpha_{2} D\right), \\
& \grave{P} \grave{S}=2 \rho_{1} \frac{\cos i_{1}}{\alpha_{1}} H p \alpha_{1} /\left(\beta_{2} D\right), \\
& \grave{S} \dot{P}=-2 \frac{\cos j_{1}}{\beta_{1}}\left(a b+c d \frac{\cos i_{2}}{\alpha_{2}} \frac{\cos j_{2}}{\beta_{2}}\right) p \beta_{1} /\left(\alpha_{1} D\right), \\
& \grave{S} \dot{S}=-\left[\left(b \frac{\cos j_{1}}{\beta_{1}}-c \frac{\cos j_{2}}{\beta_{2}}\right) E-\left(a+d \frac{\cos i_{2}}{\alpha_{2}} \frac{\cos j_{1}}{\beta_{1}}\right) G p^{2}\right] / D \\
& \grave{S} \grave{P}=-2 \rho_{1} \frac{\cos j_{1}}{\beta_{1}} G p \beta_{1} /\left(\alpha_{2} D\right), \\
& \grave{S} \grave{S}=2 \rho_{1} \frac{\cos j_{1}}{\beta_{1}} E \beta_{1} /\left(\beta_{2} D\right),
\end{aligned}
$$

where $\alpha_{k}=\sqrt{\left(\lambda_{k}+2 \mu_{k}\right) / \rho_{k}}, \beta_{k}=\sqrt{\mu_{k} / \rho_{k}}, k=1,2$ which indicates the different materials around the interface. $i_{1}, i_{2}$ stand for angles of incident and transmitted Pwaves; $j_{1}, j_{2}$ stand for angles of incident and transmitted S-waves, $p=(\sin i) / \alpha=$ $(\sin j) / \beta$ is called ray parameter, a parameter of a whole system of rays. Repeatedly used variables $a, b, c, d, E, F, G, H, D$ are given by:

$$
\begin{aligned}
a & =\rho_{2}\left(1-2 \beta_{2}^{2} p^{2}\right)-\rho_{1}\left(1-2 \beta_{1}^{2} p^{2}\right), & b & =\rho_{2}\left(1-2 \beta_{2}^{2} p^{2}\right)+2 \rho_{1} \beta_{1}^{2} p^{2}, \\
c & =\rho_{1}\left(1-2 \beta_{1}^{2} p^{2}\right)+2 \rho_{2} \beta_{2}^{2} p^{2}, & d & =2\left(\rho_{2} \beta_{2}^{2}-\rho_{1} \beta_{1}^{2}\right),
\end{aligned}
$$


and

$$
\begin{array}{ll}
E=b \frac{\cos i_{1}}{\alpha_{1}}+c \frac{\cos i_{2}}{\alpha_{2}}, & F=b \frac{\cos j_{1}}{\beta_{1}}+c \frac{\cos j_{2}}{\beta_{2}}, \\
G=a-d \frac{\cos i_{1}}{\alpha_{1}} \frac{\cos j_{2}}{\beta_{2}}, & H=a-d \frac{\cos i_{2}}{\alpha_{2}} \frac{\cos j_{1}}{\beta_{1}}, \\
D=E F+G H p^{2} . &
\end{array}
$$

The expressions for the next two column of (2.9) can be found symmetrically.

The reflection and transmission coefficients presented above were for displacement. It follows that the energy flux satisfies:

$$
\begin{aligned}
\rho_{1} \alpha_{1} \cos i_{1}= & \rho_{1} \alpha_{1} \cos i_{1}(\grave{P} \dot{P})^{2}+\rho_{1} \beta_{1} \cos j_{1}(\grave{P} \dot{S})^{2} \\
& +\rho_{2} \alpha_{2} \cos i_{2}(\grave{P} \grave{P})^{2}+\rho_{2} \beta_{2} \cos j_{2}(\grave{P} \grave{S})^{2}
\end{aligned}
$$

for the scattered wave system given by the first column of (2.9) and it is similar for the rest of it. So we put all the energy coefficients in the following matrix:

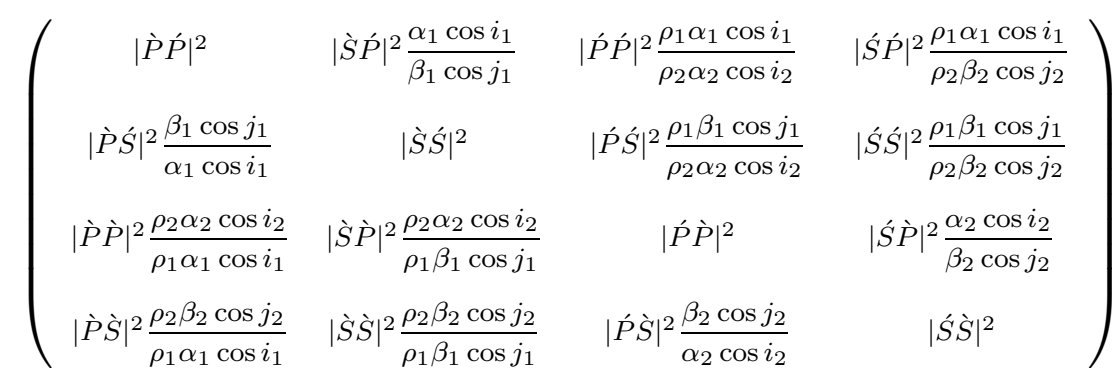

where the entries of each column will be added up to one and it displays the partition of the energy as the wave splits. In the rest part of this paper, we always use the notation $\beta_{R}^{P P}, \beta_{R}^{P S}, \beta_{T}^{P P}, \beta_{T}^{P S}$ to present the energy coefficients for the scattering of $\mathrm{P}$-wave (notation is similar for $\mathrm{S}$-wave) in the first column of (2.9) or the third, which depends on the direction of incidence. Here, $\beta_{R}^{P P}$ is the reflection coefficient from a $\mathrm{P}$-wave to a $\mathrm{P}$-wave, for example.

\section{High frequency approximation for elastic wave in phase space}

\subsection{Approximation from the hyperbolic system}

To study the high frequency approximation for elastic wave, introduce the following new variables:

$$
p=\lambda \operatorname{div} \mathbf{u}, \quad \xi_{i}=\dot{u}_{i}, \quad \epsilon_{i j}=\mu\left(\frac{\partial u_{i}}{\partial x_{j}}+\frac{\partial u_{j}}{\partial x_{i}}\right), \quad i, j=1,2 .
$$


So (2.8) becomes:

$$
\begin{aligned}
\rho \dot{\xi}_{i} & =\frac{\partial p}{\partial x_{i}}+\sum_{j} \frac{\partial \epsilon_{i j}}{\partial x_{j}} \\
\dot{\epsilon}_{i j} & =\mu\left(\frac{\partial \xi_{i}}{\partial x_{j}}+\frac{\partial \xi_{j}}{\partial x_{i}}\right) \\
\dot{p} & =\lambda \operatorname{div} \boldsymbol{\xi}
\end{aligned}
$$

Let $\mathbf{w}=\left(\xi_{1}, \xi_{2}, \epsilon_{11}, \epsilon_{22}, \epsilon_{12}, p\right),(3.1)$ can be written as a symmetric hyperbolic system

$$
A(\mathbf{x}) \frac{\partial \mathbf{w}}{\partial t}+\sum_{i} D_{i} \frac{\partial \mathbf{w}}{\partial x_{i}}=0
$$

with initial data

$$
\mathbf{w}(0, \mathbf{x})=\mathbf{w}_{0}(\mathbf{x})
$$

where the $6 \times 6$ matrix $A(\mathbf{x})=\operatorname{diag}(\rho, \rho, 1 / 2 \mu, 1 / 2 \mu, 1 / \mu, 1 / \lambda)$ and $D_{1}, D_{2}$ are constant and symmetric with entries either 0 or 1 . Define the dispersion matrix $L(\mathbf{x}, \mathbf{k})$ by

$$
\begin{aligned}
L(\mathbf{x}, \mathbf{k}) & =A^{-1}(\mathbf{x}) \sum_{i} k_{i} D_{i} \\
& =-\left(\begin{array}{cccccc}
0 & 0 & k_{1} / \rho & 0 & k_{2} / \rho & k_{1} / \rho \\
0 & 0 & 0 & k_{2} / \rho & k_{1} / \rho & k_{2} / \rho \\
2 \mu k_{1} & 0 & 0 & 0 & 0 & 0 \\
0 & 2 \mu k_{2} & 0 & 0 & 0 & 0 \\
\mu k_{2} & \mu k_{1} & 0 & 0 & 0 & 0 \\
\lambda k_{1} & \lambda k_{2} & 0 & 0 & 0 & 0
\end{array}\right)
\end{aligned}
$$

Its eigenvalues are:

$$
\begin{aligned}
& \omega_{0}=0 \text { with multiplicity two, } \\
& \omega_{ \pm}^{p}= \pm c^{p}|\mathbf{k}|, \quad \omega_{ \pm}^{s}= \pm c^{s}|\mathbf{k}|
\end{aligned}
$$

with the corresponding longitudinal and shear speeds given by (2.6) and (2.7).

In the high frequency regime, we assume that the coefficients of the matrix $A(\mathbf{x})$ vary on a scale much longer than the scale on which the initial data vary. Let $\epsilon$ be the ratio of these two scales. Rescaling space and time coordinates $(\mathbf{x}, t)$ by $\mathbf{x} \rightarrow \epsilon \mathbf{x}$, $t \rightarrow \epsilon t,(3.2)$ and (3.3) become:

$$
\begin{gathered}
A(\mathbf{x}) \frac{\partial \mathbf{w}_{\epsilon}}{\partial t}+\sum_{i} D_{i} \frac{\partial \mathbf{w}_{\epsilon}}{\partial x_{i}}=0 \\
\mathbf{w}_{\epsilon}(0, \mathbf{x})=\mathbf{w}_{0}\left(\frac{\mathbf{x}}{\epsilon}\right) \text { or } \quad \mathbf{w}_{0}\left(\frac{\mathbf{x}}{\epsilon}, \mathbf{x}\right) .
\end{gathered}
$$


Note that the parameter $\epsilon$ does not appear explicitly in (3.6). It enters through the initial condition (3.7). Particularly, we are interested in the initial data of the standard geometrical optics form

$$
\mathbf{u}_{\epsilon}(0, \mathbf{x})=\mathbf{A}_{0}(\mathbf{x}) \mathbf{e}^{i \frac{S_{0}(\mathbf{x})}{\epsilon}} .
$$

Correspondingly we will have the initial data (3.7) for (3.6). We will discuss the detailed form of this initial data later for the use of our numerical computation.

Following [22], the high frequency limit can be studied using the Wigner distribution matrix $W^{\epsilon}$ :

$$
W^{\epsilon}(t, \mathbf{x}, \mathbf{k})=\left(\frac{1}{2 \pi}\right)^{n} \int e^{i \mathbf{k} \cdot \mathbf{y}} \mathbf{w}_{\epsilon}(t, \mathbf{x}-\epsilon \mathbf{y} / 2) \mathbf{w}_{\epsilon}^{*}(t, \mathbf{x}+\epsilon \mathbf{y} / 2) d \mathbf{y}
$$

where $n$ is the space dimension and $\mathbf{w}^{*}=\overline{\mathbf{w}}^{t}$ is the conjugate transpose of $\mathbf{w}$. Although $W^{\epsilon}$ is not positive definite, it becomes so in the high frequency limit as $\epsilon \rightarrow 0$.

Let

$$
W^{(0)}(t, \mathbf{x}, \mathbf{k})=\lim _{\epsilon \rightarrow 0} W^{\epsilon}(t, \mathbf{x}, \mathbf{k}) .
$$

Since the dispersion matrix $L(\mathbf{x}, \mathbf{k})$ is self-adjoint with respect to the inner product $<$ ,$>_{A}$ i.e. $<L \mathbf{u}, \mathbf{v}>_{A}=<\mathbf{u}, L \mathbf{v}>_{A}$, all its eigenvalues $\omega_{\tau}$ are real and the corresponding eigenvectors $\mathbf{b}^{\tau}$ can be chosen to be orthogonal with respect to $<,>_{A}$ :

$$
L(\mathbf{x}, \mathbf{k}) \mathbf{b}^{\tau}(\mathbf{x}, \mathbf{k})=\omega_{\tau}(\mathbf{x}, \mathbf{k}) \mathbf{b}^{\tau}(\mathbf{x}, \mathbf{k}), \quad<\mathbf{b}^{\tau}, \mathbf{b}^{\beta}>_{A}=\delta_{\tau \beta} .
$$

For the simple eigenvalue $\omega_{\tau}(\mathbf{x}, \mathbf{k})$, one defines the matrices $B^{\tau}(\mathbf{x}, \mathbf{k})$ by

$$
B^{\tau}(\mathbf{x}, \mathbf{k})=\mathbf{b}^{\tau}(\mathbf{x}, \mathbf{k}) \mathbf{b}^{\tau *}(\mathbf{x}, \mathbf{k})
$$

so the limiting Wigner matrix $W^{(0)}(t, \mathbf{x}, \mathbf{k})$ has the form

$$
W^{(0)}(t, \mathbf{x}, \mathbf{k})=\sum_{\tau=1}^{m} a^{\tau}(t, \mathbf{x}, \mathbf{k}) B^{\tau}(\mathbf{x}, \mathbf{k})
$$

and the scalar function $a^{\tau}(t, \mathbf{x}, \mathbf{k})$ determined by projection:

$$
a^{\tau}=\operatorname{Tr}\left(A W^{(0) *} A B^{\tau}\right)
$$

satisfying the Liouville equations in the phase space [22]:

$$
\frac{\partial a^{\tau}}{\partial t}+\nabla_{\mathbf{k}} \omega_{\tau} \cdot \nabla_{\mathbf{x}} a^{\tau}-\nabla_{\mathbf{x}} \omega_{\tau} \cdot \nabla_{\mathbf{k}} a^{\tau}=0
$$

In two space dimension, $n=2$, the eigenvalues in (3.5) have corresponding basis of eigenvectors $\mathbf{b}_{ \pm}^{p}, \mathbf{b}_{ \pm}^{s}, \mathbf{b}^{0 j}, j=1,2$. Here the eigenvectors $\mathbf{b}^{01}, \mathbf{b}^{02}$ correspond to the nonpropagating modes because $\omega_{01}=\omega_{02}=0$. The eigenvectors $\mathbf{b}_{ \pm}^{p}$ represent the forward and the backward longitudinal modes, the P-waves, which propagate with the wave speed $c^{p}$. The eigenvectors $\mathbf{b}_{ \pm}^{s}$ represent forward and backward transverse modes, the 
S-waves, which propagate with the wave speed $c^{s}$. Hence without loss of generality, we can neglect the non-propagating modes and write the limit Wigner distribution matrix $W^{(0)}(t, \mathbf{x}, \mathbf{k})$ as

$$
\begin{aligned}
W^{(0)}(t, \mathbf{x}, \mathbf{k})= & a_{+}^{p}(t, \mathbf{x}, \mathbf{k}) \mathbf{b}_{+}^{p}(\mathbf{k}) \mathbf{b}_{+}^{p *}(\mathbf{k})+a_{-}^{p}(t, \mathbf{x}, \mathbf{k}) \mathbf{b}_{-}^{p}(\mathbf{k}) \mathbf{b}_{-}^{p *}(\mathbf{k}) \\
& +a_{+}^{s}(t, \mathbf{x}, \mathbf{k}) \mathbf{b}_{+}^{s}(\mathbf{k}) \mathbf{b}_{+}^{s *}(\mathbf{k})+a_{-}^{s}(t, \mathbf{x}, \mathbf{k}) \mathbf{b}_{-}^{s}(\mathbf{k}) \mathbf{b}_{-}^{s *}(\mathbf{k})
\end{aligned}
$$

The scalar functions $a_{+}^{p}$ and $a_{-}^{p}$ are related by $a_{+}^{p}(t, \mathbf{x}, \mathbf{k})=a_{-}^{p}(t, \mathbf{x},-\mathbf{k})$ and similar for $a_{ \pm}^{s}$. They satisfy the Liouville equation :

$$
\begin{aligned}
& \frac{\partial a_{+}^{p}}{\partial t}+c^{p}(\mathbf{x}) \hat{\mathbf{k}} \cdot \nabla_{\mathbf{x}} a_{+}^{p}-|\mathbf{k}| \nabla_{\mathbf{x}} c^{p}(\mathbf{x}) \cdot \nabla_{\mathbf{k}} a_{+}^{p}=0 \\
& \frac{\partial a_{+}^{s}}{\partial t}+c^{s}(\mathbf{x}) \hat{\mathbf{k}} \cdot \nabla_{\mathbf{x}} a_{+}^{s}-|\mathbf{k}| \nabla_{\mathbf{x}} c^{s}(\mathbf{x}) \cdot \nabla_{\mathbf{k}} a_{+}^{s}=0 .
\end{aligned}
$$

The spatial energy density for the solutions of (3.6) is given by

$$
\begin{aligned}
E^{\epsilon}(t, \mathbf{x}) & =\frac{1}{2}\left(A(\mathbf{x}) \mathbf{w}_{\epsilon}(t, \mathbf{x}), \mathbf{w}_{\epsilon}(t, \mathbf{x})\right) \\
& =\frac{1}{2} \int \operatorname{Tr}\left(A(\mathbf{x}) W^{\epsilon}(t, \mathbf{x}, \mathbf{k})\right) d \mathbf{k}
\end{aligned}
$$

and the energy flux by

$$
\begin{aligned}
F_{i}^{\epsilon}(t, \mathbf{x}) & =\frac{1}{2}\left(D_{i} \mathbf{w}_{\epsilon}(t, \mathbf{x}), \mathbf{w}_{\epsilon}(t, \mathbf{x})\right) \\
& =\frac{1}{2} \int \operatorname{Tr}\left(D_{i} W^{\epsilon}(t, \mathbf{x}, \mathbf{k})\right) d \mathbf{k} .
\end{aligned}
$$

When $\epsilon \rightarrow 0$, the high frequency limit of $E^{\epsilon}(t, \mathbf{x})$ and $\mathbf{F}^{\epsilon}(t, \mathbf{x})$ are :

$$
\begin{aligned}
E(t, \mathbf{x}) & =\frac{1}{2} \int \operatorname{Tr}\left(A(\mathbf{x}) W^{(0)}(t, \mathbf{x}, \mathbf{k})\right) d \mathbf{k} \\
& =\int\left(a_{+}^{p}(t, \mathbf{x}, \mathbf{k})+a_{+}^{s}(t, \mathbf{x}, \mathbf{k})\right) d \mathbf{k}
\end{aligned}
$$

and

$$
\mathbf{F}(t, \mathbf{x})=\int \hat{\mathbf{k}}\left(c^{p}(\mathbf{x}) a_{+}^{p}(t, \mathbf{x}, \mathbf{k})+c^{s}(\mathbf{x}) a_{+}^{s}(t, \mathbf{x}, \mathbf{k})\right) d \mathbf{k} .
$$

Therefore, $a_{+}^{p}$ and $a_{+}^{s}$ can be interpreted as phase space energy densities (their nonnegativity can be obtained from the non-negativity of $\left.W^{(0)}\right)$ and we aim at computing their zeroth moments which are summed to be the spatial energy density $E(t, \mathbf{x})$ after we solve the Liouville equation (3.14) and (3.15). Note that (3.14) and (3.15) are decupled in homogeneous media.

The computational challenge arises with discontinuous wave speed $c^{p}$ and $c^{s}$, corresponding to non-homogeneous media where the two Liouville equations (3.14) and 
(3.15) are coupled due to wave modes conversion at the interface. Moreover, these Liouville equations have singular (discontinuous and measure-valued) coefficients at the interface.

In the simple case of a flat interface and homogeneous media adjacent to the interface, we follow the ideas $[10,12]$, where the behavior of waves at the interface are built into the numerical flux. This gives a numerically efficient way to solve (3.14) and (3.15) even with the discontinuous $c^{p}$ and $c^{s}$.

\subsection{Approximation by WKB expansion}

A classical way to study the high frequency limit is the WKB analysis. Consider (2.8) in the homogeneous media and it can be written in the vector form

$$
\rho \frac{\partial^{2} \mathbf{u}}{\partial t^{2}}=(\lambda+\mu) \nabla(\nabla \cdot \mathbf{u})+\mu \nabla^{2} \mathbf{u}
$$

with the geometrical optics initial data (3.8). We look for a solution of (3.20) in the form

$$
\mathbf{u}(t, \mathbf{x})=\mathbf{A}(t, \mathbf{x}) \mathbf{e}^{i \frac{S(t, \mathbf{x})}{\epsilon}}
$$

with

$$
\mathbf{A}(t, \mathbf{x})=\mathbf{A}_{0}(t, \mathbf{x})+\epsilon \mathbf{A}_{1}(t, \mathbf{x})+\epsilon^{2} \mathbf{A}_{2}(t, \mathbf{x})+\cdots .
$$

Substituting the expression (3.21) into (3.20), one obtains

$$
\begin{gathered}
\rho\left(\frac{\partial^{2} \mathbf{A}}{\partial t^{2}}+2 \frac{i}{\epsilon} \frac{\partial \mathbf{A}}{\partial t} \frac{\partial S}{\partial t}+\frac{i}{\epsilon} \mathbf{A} \frac{\partial^{2} S}{\partial t^{2}}+\left(\frac{i}{\epsilon}\right)^{2} \mathbf{A}\left(\frac{\partial S}{\partial t}\right)^{2}\right) \\
=(\lambda+\mu)\left(\nabla(\nabla \cdot \mathbf{A})+\frac{i}{\epsilon}(\nabla \cdot \mathbf{A}) \nabla S+\frac{i}{\epsilon} \nabla(\mathbf{A} \cdot \nabla S)+\left(\frac{i}{\epsilon}\right)^{2}(\mathbf{A} \cdot \nabla S) \nabla S\right) \\
+\mu\left(\nabla^{2} \mathbf{A}+2 \frac{i}{\epsilon} \nabla \mathbf{A} \nabla S+\frac{i}{\epsilon} \mathbf{A} \nabla^{2} S+\left(\frac{i}{\epsilon}\right)^{2} \mathbf{A}|\nabla S|^{2}\right) .
\end{gathered}
$$

We now balance coefficients of $\epsilon$ in (3.22). The leading order $O\left(\epsilon^{-2}\right)$ is :

$$
\rho \mathbf{A}_{0}\left(\frac{\partial S}{\partial t}\right)^{2}=(\lambda+\mu)\left(\mathbf{A}_{0} \cdot \nabla S\right) \nabla S+\mu \mathbf{A}_{0}|\nabla S|^{2}
$$

while the next term $O\left(\epsilon^{-1}\right)$ is :

$$
\begin{aligned}
2 \rho \frac{\partial \mathbf{A}_{0}}{\partial t} \frac{\partial S}{\partial t}+\rho \mathbf{A}_{0} \frac{\partial^{2} S}{\partial t^{2}}= & (\lambda+\mu)\left(\left(\nabla \cdot \mathbf{A}_{0}\right) \nabla S+\nabla\left(\mathbf{A}_{0} \cdot \nabla S\right)\right) \\
& +\mu\left(2 \nabla \mathbf{A}_{0} \nabla S+\mathbf{A}_{0} \nabla^{2} S\right)
\end{aligned}
$$

Taking inner product with the vector $\nabla S$ on both side of (3.23) yields :

$$
\left(\mathbf{A}_{0} \cdot \nabla S\right)\left(\rho\left(\frac{\partial S}{\partial t}\right)^{2}-(\lambda+2 \mu)|\nabla S|^{2}\right)=0
$$


Analogous to (2.5), (3.25) will be satisfied in two ways:

Case 1 : If $\mathbf{A}_{0} \cdot \nabla S=0,(3.23)$ becomes

$$
\rho \mathbf{A}_{0}\left(\frac{\partial S}{\partial t}\right)^{2}=\mu \mathbf{A}_{0}|\nabla S|^{2}
$$

which gives the eikonal equations

$$
\partial_{t} S \pm c^{s}|\nabla S|=0
$$

This case just corresponds to the transverse wave, i.e. the motion $\left(\mathbf{A}_{0}\right)$ is normal to the direction of propagation $(\nabla S)$.

Case 2 : If $\rho\left(\partial_{t} S\right)^{2}-(\lambda+2 \mu)|\nabla S|^{2}=0$, then

$$
\partial_{t} S \pm c^{p}|\nabla S|=0
$$

substituting (3.27) into (3.23) yields

$$
\mathbf{A}_{0}|\nabla S|^{2}=\left(\mathbf{A}_{0} \cdot \nabla S\right) \nabla S
$$

which denotes $\mathbf{A}_{0}=a \nabla S$ where $a$ is a scalar. So this case corresponds to the longitudinal wave, i.e. the motion $\left(\mathbf{A}_{0}\right)$ is parallel to the direction of propagation $(\nabla S)$.

In the first case, taking the inner product with vector $\mathbf{A}_{0}$ on both sides of (3.24) :

$$
\rho \frac{\partial\left|\mathbf{A}_{0}\right|^{2}}{\partial t} \frac{\partial S}{\partial t}+\rho\left|\mathbf{A}_{0}\right|^{2} \frac{\partial^{2} S}{\partial t^{2}}=\mu \nabla \cdot\left(\left|\mathbf{A}_{0}\right|^{2} \nabla S\right)
$$

using (3.26) with the minus sign we can simplify (3.28) into

$$
\frac{\partial\left|\mathbf{A}_{0}\right|^{2}}{\partial t}-\left(c^{s}\right)^{2} \nabla_{\mathbf{x}} \cdot\left(\left|\mathbf{A}_{0}\right|^{2} \frac{\nabla S}{c^{s}|\nabla S|}\right)=0 .
$$

Let $\mathcal{E}^{s}=\left|\frac{\mathbf{A}_{0}}{c^{s}}\right|^{2}$, then $(3.29)$ can be written as

$$
\frac{\partial \mathcal{E}^{s}}{\partial t}+\nabla_{\mathbf{x}} \cdot\left(\mathcal{E}^{s} \nabla_{\mathbf{k}} H_{s}(\mathbf{x}, \nabla S)\right)=0
$$

with $H_{s}(\mathbf{x}, \mathbf{k})=-c^{s}(\mathbf{x})|\mathbf{k}|$. If we use (3.26) with the plus sign, the S-wave energy density $\mathcal{E}^{s}$ still satisfies the above equation except for $H_{s}(\mathbf{x}, \mathbf{k})=c^{s}(\mathbf{x})|\mathbf{k}|$.

Similarly in the second case, taking the inner product with vector $\mathbf{A}_{0}$ on both sides of $(3.24)$ :

$$
\rho \frac{\partial\left|\mathbf{A}_{0}\right|^{2}}{\partial t} \frac{\partial S}{\partial t}+\rho\left|\mathbf{A}_{0}\right|^{2} \frac{\partial^{2} S}{\partial t^{2}}=(\lambda+2 \mu) \nabla \cdot\left(\left|\mathbf{A}_{0}\right|^{2} \nabla S\right)
$$

we can also get the conservative transport equation for the P-wave by inserting (3.27) into (3.31) :

$$
\frac{\partial \mathcal{E}^{p}}{\partial t}+\nabla_{\mathbf{x}} \cdot\left(\mathcal{E}^{p} \nabla_{\mathbf{k}} H_{p}(\mathbf{x}, \nabla S)\right)=0
$$


with $\mathcal{E}^{p}=\left|\frac{\mathbf{A}_{0}}{c^{p}}\right|^{2}$ and $H_{p}(\mathbf{x}, \mathbf{k})= \pm c^{p}(\mathbf{x})|\mathbf{k}|$.

Without loss of generality, we consider the eikonal and transport equations (3.27), (3.32) for P-wave and (3.26), (3.30) for S-wave with positive Hamiltonians. They can also be derived from (3.14) and (3.15) as follows. The solution of the form (3.21) for (3.20) implies that the initial condition (3.7) for (3.6) can be written into the similar form

$$
\mathbf{W}_{\epsilon}(0, \mathbf{x})=\mathbf{B}_{0}^{\epsilon}(\mathbf{x}) \mathbf{e}^{i \frac{S_{0}(\mathbf{x})}{\epsilon}}
$$

where $\mathbf{B}_{0}^{\epsilon}$ is the new amplitude in terms of $\epsilon, \mathbf{A}_{0}, S_{0}$ and their derivatives.

Applying (3.33) in (3.9):

$$
\begin{array}{r}
W^{\epsilon}(0, \mathbf{x}, \mathbf{k})=\left(\frac{1}{2 \pi}\right)^{n} \int e^{i \mathbf{k} \cdot \mathbf{y}} \mathbf{B}_{0}^{\epsilon}(\mathbf{x}-\epsilon \mathbf{y} / 2) \mathbf{B}_{0}^{\epsilon *}(\mathbf{x}+\epsilon \mathbf{y} / 2) . \\
\mathbf{e}^{i\left(S_{0}(\mathbf{x}-\epsilon \mathbf{y} / 2)-S_{0}(\mathbf{x}+\epsilon \mathbf{y} / 2)\right) / \epsilon} d \mathbf{y}
\end{array}
$$

The weak limit of $W^{\epsilon}(0, \mathbf{x}, \mathbf{k})$, in the sense of distribution, is

$$
W^{(0)}(0, \mathbf{x}, \mathbf{k})=\mathbf{B}_{0}(\mathbf{x}) \mathbf{B}_{0}^{*}(\mathbf{x}) \boldsymbol{\delta}\left(\mathbf{k}-\nabla S_{0}(\mathbf{x})\right)
$$

and it follows by (3.11) that

$$
\begin{aligned}
a_{+}^{\tau}(0, \mathbf{x}, \mathbf{k}) & =\operatorname{Tr}\left(A \mathbf{B}_{0} \mathbf{B}_{0}^{*} A B_{+}^{\tau}\right) \boldsymbol{\delta}\left(\mathbf{k}-\nabla S_{0}(\mathbf{x})\right) \\
& \doteq \mathcal{E}_{0}^{\tau}(\mathbf{x}) \boldsymbol{\delta}\left(\mathbf{k}-\nabla S_{0}(\mathbf{x})\right), \quad \tau=p, s .
\end{aligned}
$$

Let the functions $S^{p}(t, \mathbf{x})$ and $\mathcal{E}^{p}(t, \mathbf{x})$ be the solutions of the eikonal and transport equations (3.27) and (3.32) with the initial conditions $S^{p}(0, \mathbf{x})=S_{0}(\mathbf{x})$ and $\mathcal{E}^{p}(0, \mathbf{x})=\mathcal{E}_{0}^{p}(\mathbf{x})$; $S^{s}(t, \mathbf{x})$ and $\mathcal{E}^{s}(t, \mathbf{x})$ be the solutions of (3.26) and (3.30) with the initial conditions $S^{s}(0, \mathbf{x})=S_{0}(\mathbf{x})$ and $\mathcal{E}^{s}(0, \mathbf{x})=\mathcal{E}_{0}^{s}(\mathbf{x})$. Then the solutions of equation (3.14) and (3.15) before the formation of caustics, are

$$
a_{+}^{\tau}(t, \mathbf{x}, \mathbf{k})=\mathcal{E}^{\tau}(t, \mathbf{x}) \boldsymbol{\delta}\left(\mathbf{k}-\nabla S^{\tau}(t, \mathbf{x})\right), \quad \tau=p, s
$$

Conversely, given initial conditions of the form (3.35) for (3.14) and (3.15) and $\left\{a_{+}^{\tau}, \tau=\right.$ $p, s\}$ given by (3.36), then $S^{\tau}$ and $\mathcal{E}^{\tau}$ must satisfy the eikonal and transport equations respectively by taking the zeroth and first moment of the Liouville equations (3.14) and (3.15). This shows that we can recover from the Liouville equation the usual WKB approximation. However, the WKB analysis is no longer valid after the formation of caustics, since a calculation based on the eikonal equation and the transport equation picks up the so-called viscosity solution, while the physical solution to the eikonal and transport equations becomes multi-valued. Thus we need to solve the Liouville equation (3.14) and (3.15) which are valid even beyond caustics. 


\section{Numerical scheme for the Liouville equations with discontinuous wave speed}

\subsection{Preliminary}

Consider the 2D Liouville equation for the P-wave and the S-wave:

$$
\begin{aligned}
& \frac{\partial a^{p}}{\partial t}+c^{p}(\mathbf{x}) \hat{\mathbf{k}} \cdot \nabla_{\mathbf{x}} a^{p}-|\mathbf{k}| \nabla_{\mathbf{x}} c^{p}(\mathbf{x}) \cdot \nabla_{\mathbf{k}} a^{p}=0 \\
& \frac{\partial a^{s}}{\partial t}+c^{s}(\mathbf{x}) \hat{\mathbf{k}} \cdot \nabla_{\mathbf{x}} a^{s}-|\mathbf{k}| \nabla_{\mathbf{x}} c^{s}(\mathbf{x}) \cdot \nabla_{\mathbf{k}} a^{s}=0
\end{aligned}
$$

with the initial condition

$$
\begin{aligned}
& a^{p}(0, \mathbf{x}, \mathbf{k})=\mathcal{E}_{0}^{p}(\mathbf{x}) \boldsymbol{\delta}\left(\mathbf{k}-\nabla S_{0}\right) \\
& a^{s}(0, \mathbf{x}, \mathbf{k})=\mathcal{E}_{0}^{s}(\mathbf{x}) \boldsymbol{\delta}\left(\mathbf{k}-\nabla S_{0}\right)
\end{aligned}
$$

where $\mathbf{x}=(x, y), \mathbf{k}=(\xi, \eta), \hat{\mathbf{k}}=\left(\frac{\xi}{\sqrt{\xi^{2}+\eta^{2}}}, \frac{\eta}{\sqrt{\xi^{2}+\eta^{2}}}\right)$.

When $c^{p}(\mathbf{x})$ and $c^{s}(\mathbf{x})$ are discontinuous, the Liouville equations (4.1) and (4.2) have discontinuity and measure-valued coefficients. Here we extend the Hamiltonianpreserving scheme in [11] and [12] to solve (4.1) and (4.2) with discontinuous wave speeds $c^{p}$ and $c^{s}$. When a $\mathrm{P}$-wave moves with its density distribution governed by the Liouville equation (4.1) or a S-wave governed by the Liouville equation (4.2), their Hamiltonian $H=c|\mathbf{k}|$ should be preserved across the interface:

$$
c^{p-}\left|\mathbf{k}^{p-}\right|=c^{s-}\left|\mathbf{k}^{s-}\right|=c^{p+}\left|\mathbf{k}^{p+}\right|=c^{s+}\left|\mathbf{k}^{s+}\right|
$$

where the superscripts \pm indicates the right and left limits of the quantity at the interface. This is due to the fact that $\mathrm{P}$-wave and $\mathrm{S}$-wave can convert to each other with energy conserved when they hit the interface. How the energy splits between these two waves is shown in Section 2.2. The condition (4.5) can be used to determine the wave number $k$ on one side of the interface from its value on the other side. In details, we take the P-wave as an example:

- When a plane incident P-wave hits a vertical interface:

Let $\mathbf{x}=(x, y), \mathbf{k}=(\xi, \eta)$. Assume the incident wave has wave number $\left(\xi^{-}, \eta^{-}\right)$ to the left side of the interface, with $\xi^{-}>0$. Since the interface is vertical, the bi-characteristics of the Liouville equation (4.1) implies that $\eta$ is not changed when the wave crosses the interface. There are four possible splitting waves:

1. There is absolutely a reflected $\mathrm{P}$-wave with $\beta_{R}^{P P}$ proportion of the energy that has the wave number $\left(-\xi^{-}, \eta^{-}\right)$. 
2. If $\left(\frac{c^{p-}}{c^{p+}}\right)^{2}\left(\xi^{-}\right)^{2}+\left[\left(\frac{c^{p-}}{c^{p+}}\right)^{2}-1\right]\left(\eta^{-}\right)^{2}>0$, the wave can partially transmit to a P-wave, with $\beta_{T}^{P P}$ proportion of the energy. The new wave number will be $\left(\xi^{p p+}, \eta^{-}\right)$where

$$
\xi^{p p+}=\sqrt{\left(\frac{c^{p-}}{c^{p+}}\right)^{2}\left(\xi^{-}\right)^{2}+\left[\left(\frac{c^{p-}}{c^{p+}}\right)^{2}-1\right]\left(\eta^{-}\right)^{2}}
$$

is obtained by (4.5). Otherwise, the wave number becomes imaginary and this kind of wave is called evanescent wave or inhomogeneous wave [2],[4]. In the high frequency limit, the energy in evanescent waves is exponentially small and vanishes as $\epsilon \rightarrow 0$ [3]. So there is no transmitted P-wave according to the energy partition and we can set $\beta_{T}^{P P}=0$.

3. If $\left(\frac{c^{p-}}{c^{s+}}\right)^{2}\left(\xi^{-}\right)^{2}+\left[\left(\frac{c^{p-}}{c^{s+}}\right)^{2}-1\right]\left(\eta^{-}\right)^{2}>0$, the wave can partially transmit to a S-wave, with $\beta_{T}^{P S}$ proportion of the energy. The new wave number will be $\left(\xi^{p s+}, \eta^{-}\right)$where

$$
\xi^{p s+}=\sqrt{\left(\frac{c^{p-}}{c^{s+}}\right)^{2}\left(\xi^{-}\right)^{2}+\left[\left(\frac{c^{p-}}{c^{s+}}\right)^{2}-1\right]\left(\eta^{-}\right)^{2}} .
$$

Otherwise, similar to Case 2, there is no transmitted S-wave according to the energy partition and we can set $\beta_{T}^{P S}=0$.

4. If $\left(\frac{c^{p-}}{c^{s-}}\right)^{2}\left(\xi^{-}\right)^{2}+\left[\left(\frac{c^{p-}}{c^{s-}}\right)^{2}-1\right]\left(\eta^{-}\right)^{2}>0$, the wave can partially reflect to a S-wave, with $\beta_{R}^{P S}$ proportion of the energy. The new wave number will be $\left(\xi^{p s-}, \eta^{-}\right)$where

$$
\xi^{p s-}=\sqrt{\left(\frac{c^{p-}}{c^{s-}}\right)^{2}\left(\xi^{-}\right)^{2}+\left[\left(\frac{c^{p-}}{c^{s-}}\right)^{2}-1\right]\left(\eta^{-}\right)^{2}} .
$$

Otherwise, there is no reflected S-wave according to the energy partition and we can set $\beta_{R}^{P S}=0$.

For all these four possible splitting waves, the energy coefficients $\beta_{R}^{P P}, \beta_{R}^{P S}, \beta_{T}^{P P}$, $\beta_{T}^{P S}$ can be obtained by the way described in Section 2.2. They always satisfy:

$$
\beta_{R}^{P P}+\beta_{R}^{P S}+\beta_{T}^{P P}+\beta_{T}^{P S}=1 .
$$

When some of the wave numbers become imaginary and the associated energy becomes zero, the total energy will be partitioned among the other waves.

If $\xi^{-}<0$, similar behavior can also be analyzed using the constant Hamiltonian condition (4.5). 
Since we need to consider the mode conversion at the interface between $\mathrm{P}$-wave and S-wave, the solutions of Liouville equation (4.1) and (4.2) need to be determined from four bi-characteristics respectively. Hence, we use the following condition at the interface:

$$
\begin{aligned}
a^{p}\left(t, \mathbf{x}^{+}, \mathbf{k}^{+}\right)= & \beta_{R}^{P P} \cdot a^{p}\left(t, \mathbf{x}^{+}, \mathbf{k}_{p_{1}}\right)+\beta_{R}^{S P} \cdot a^{s}\left(t, \mathbf{x}^{+}, \mathbf{k}_{p_{2}}\right) \\
& +\beta_{T}^{P P} \cdot a^{p}\left(t, \mathbf{x}^{-}, \mathbf{k}_{p_{3}}\right)+\beta_{T}^{S P} \cdot a^{s}\left(t, \mathbf{x}^{-}, \mathbf{k}_{p_{4}}\right) \\
a^{s}\left(t, \mathbf{x}^{+}, \mathbf{k}^{+}\right)= & \beta_{R}^{P S} \cdot a^{p}\left(t, \mathbf{x}^{+}, \mathbf{k}_{s_{1}}\right)+\beta_{R}^{S S} \cdot a^{s}\left(t, \mathbf{x}^{+}, \mathbf{k}_{s_{2}}\right) \\
& +\beta_{T}^{P S} \cdot a^{p}\left(t, \mathbf{x}^{-}, \mathbf{k}_{s_{3}}\right)+\beta_{T}^{S S} \cdot a^{s}\left(t, \mathbf{x}^{-}, \mathbf{k}_{s_{4}}\right)
\end{aligned}
$$

where $\mathbf{k}_{p_{i}}, \mathbf{k}_{s_{i}}, i=1, \cdots, 4$ are the new wave numbers determined through the constant Hamiltonian condition (4.5).

Note that the Liouville equations (4.1) and (4.2) are linear hyperbolic equations with discontinuous and measure-valued coefficients when $c^{p}$ and $c^{s}$ contain discontinuity. Such a problem is not well-posed in the classical sense. Our analytic solution is given by method of characteristics away from the interface, then passing through the interface, using the interface condition (4.6). Thus we connect the two Liouville equations in "good" domains-where $c^{p}$ and $c^{s}$ are smooth-by the interface condition (4.6). The initial-boundary value problem is clearly well-posed. This interface condition will be built into the numerical flux, giving a scheme yielding the correct scattering at the interface.

\subsection{A Hamiltonian preserving scheme}

We employ a uniform mesh with grid points at $x_{i+\frac{1}{2}}, y_{j+\frac{1}{2}}, \xi_{k+\frac{1}{2}}, \eta_{l+\frac{1}{2}}$ in each direction. The cells are centered at $\left(x_{i}, y_{j}, \xi_{k}, \eta_{l}\right)$ with $x_{i}=\frac{1}{2}\left(x_{i+\frac{1}{2}}+x_{i-\frac{1}{2}}\right), y_{j}=\frac{1}{2}\left(y_{j+\frac{1}{2}}+y_{j-\frac{1}{2}}\right)$, $\xi_{k}=\frac{1}{2}\left(\xi_{k+\frac{1}{2}}+\xi_{k-\frac{1}{2}}\right), \eta_{l}=\frac{1}{2}\left(\eta_{l+\frac{1}{2}}+\eta_{l-\frac{1}{2}}\right)$. The mesh size is denoted by $\Delta x=x_{i+\frac{1}{2}}-x_{i-\frac{1}{2}}$, $\Delta y=y_{j+\frac{1}{2}}-y_{j-\frac{1}{2}}, \Delta \xi=\xi_{k+\frac{1}{2}}-\xi_{k-\frac{1}{2}}, \Delta \eta=\eta_{l+\frac{1}{2}}-\eta_{l-\frac{1}{2}}$. The cell average of $a^{\tau}, \tau=p, s$, is defined as

$$
a_{i j k l}^{\tau}=\frac{1}{\Delta x \Delta y \Delta \xi \Delta \eta} \int_{x_{i-\frac{1}{2}}}^{x_{i+\frac{1}{2}}} \int_{y_{j-\frac{1}{2}}}^{y_{j+\frac{1}{2}}} \int_{\xi_{k-\frac{1}{2}}}^{\xi_{k+\frac{1}{2}}} \int_{\eta_{l-\frac{1}{2}}}^{\eta_{l+\frac{1}{2}}} a^{\tau}(x, y, \xi, \eta) d \eta d \xi d y d x
$$

Assume that the discontinuous points of wave speed $c^{\tau}(\tau=p, s)$ are located at the grid points. Let the $x$-direction limit of $c^{\tau}(x, y)$ at point $x_{i+1 / 2}$ be $c_{i+\frac{1}{2}, j}^{\tau+}$ and $c_{i+\frac{1}{2}, j}^{\tau-}$, and the $y$-direction limit of $c^{\tau}(x, y)$ at point $y_{i+1 / 2}$ be $c_{i, j+\frac{1}{2}}^{\tau+}$ and $c_{i, j+\frac{1}{2}}^{\tau-}$. We approximate $c^{\tau}$ by a piecewise bilinear function

$$
\begin{aligned}
c^{\tau}(x, y) \approx c_{i-1 / 2, j-1 / 2}^{\tau+} & +\frac{c_{i+1 / 2, j}^{\tau-}-c_{i-1 / 2, j}^{\tau+}}{\Delta x}\left(x-x_{i-1 / 2}\right) \\
& +\frac{c_{i, j+1 / 2}^{\tau-}-c_{i, j-1 / 2}^{\tau+}}{\Delta y}\left(y-y_{i-1 / 2}\right),
\end{aligned}
$$


and define the averaged wave speed in a cell by averaging the four cell interface wave speed values

$$
c_{i j}^{\tau}=\frac{1}{4}\left(c_{i-\frac{1}{2}, j}^{\tau+}+c_{i+\frac{1}{2}, j}^{\tau-}+c_{i, j-\frac{1}{2}}^{\tau+}+c_{i, j+\frac{1}{2}}^{\tau-}\right), \quad \tau=p, s .
$$

The 2D Liouville equation (4.1) and (4.2) can be semi-discretized as

$$
\begin{aligned}
\left(a_{i j k l}^{\tau}\right)_{t} & +\frac{c_{i j}^{\tau} \xi_{k}}{\Delta x \sqrt{\xi_{k}^{2}+\eta_{l}^{2}}}\left(a_{i+\frac{1}{2}, j k l}^{\tau-}-a_{i-\frac{1}{2}, j k l}^{\tau+}\right) \\
& +\frac{c_{i j}^{\tau} \eta_{l}}{\Delta y \sqrt{\xi_{k}^{2}+\eta_{l}^{2}}}\left(a_{i, j+\frac{1}{2}, k l}^{\tau-}-a_{i, j-\frac{1}{2}, k l}^{\tau+}\right) \\
& -\frac{c_{i+\frac{1}{2}, j}^{\tau-}-c_{i-\frac{1}{2}, j}^{\tau+}}{\Delta x \Delta \xi} \sqrt{\xi_{k}^{2}+\eta_{l}^{2}}\left(a_{i j, k+\frac{1}{2}, l}^{\tau}-a_{i j, k-\frac{1}{2}, l}^{\tau}\right) \\
& -\frac{c_{i, j+\frac{1}{2}}^{\tau-}-c_{i, j-\frac{1}{2}}^{\tau+}}{\Delta y \Delta \eta} \sqrt{\xi_{k}^{2}+\eta_{l}^{2}}\left(a_{i j k, l+\frac{1}{2}}^{\tau}-a_{i j k, l-\frac{1}{2}}^{\tau}\right) \\
= & 0 \quad \text { for } \tau=p, s,
\end{aligned}
$$

where the interface values $a_{i j, k+\frac{1}{2}, l}^{\tau}, a_{i j k, l+\frac{1}{2}}^{\tau}$ are provided by the upwind approximation. Since the characteristics of the Liouville equation may be different on the two sides of the interface, the corresponding numerical fluxes should also be different. We will use (4.6) to define the split interface values $a_{i+\frac{1}{2}, j k l}^{\tau-}, a_{i-\frac{1}{2}, j k l}^{\tau+}, a_{i, j+\frac{1}{2}, k l}^{\tau-}, a_{i, j-\frac{1}{2}, k l}^{\tau+}$.

If the interface lies in the vertical direction, we can assume $c^{\tau}(x, y)$ is discontinuous at $x_{i+\frac{1}{2}}$. Consider the case $\xi_{j}>0$. Using upwind scheme, $a_{i+\frac{1}{2}, j k l}^{\tau-}=a_{i j k l}^{\tau}$ for $\tau=p, s$. However, from (4.6),

$$
\begin{aligned}
a_{i+\frac{1}{2}, j k l}^{p+}= & \beta_{R}^{P P} a^{p}\left(t, x_{i+\frac{1}{2}}^{+}, y_{j},-\xi_{k}^{+}, \eta_{l}\right)+\beta_{R}^{S P} a^{s}\left(t, x_{i+\frac{1}{2}}^{+}, y_{j}, \xi^{s p+}, \eta_{l}\right) \\
& +\beta_{T}^{P P} a^{p}\left(t, x_{i+\frac{1}{2}}^{-}, y_{j}, \xi^{p p-}, \eta_{l}\right)+\beta_{T}^{S P} a^{s}\left(t, x_{i+\frac{1}{2}}^{-}, y_{j}, \xi^{s p-}, \eta_{l}\right) \\
a_{i+\frac{1}{2}, j k l}^{s+}= & \beta_{R}^{P S} a^{p}\left(t, x_{i+\frac{1}{2}}^{+}, y_{j}, \xi^{p s+}, \eta_{l}\right)+\beta_{R}^{S S} a^{s}\left(t, x_{i+\frac{1}{2}}^{+}, y_{j},-\xi_{k}^{+}, \eta_{l}\right) \\
& +\beta_{T}^{P S} a^{p}\left(t, x_{i+\frac{1}{2}}^{-}, y_{j}, \xi^{p s-}, \eta_{l}\right)+\beta_{T}^{S S} a^{s}\left(t, x_{i+\frac{1}{2}}^{-}, y_{j}, \xi^{s s-}, \eta_{l}\right)
\end{aligned}
$$

where $\xi_{k}^{+}=\xi_{k}$ and $\xi^{s p+}, \xi^{p p-}, \xi^{s p-}, \xi^{p s+}, \xi^{p s-}, \xi^{s s-}$ are obtained from (4.5), as discussed in section 4.1. Since these new wave numbers may not be grid points, we have to define them approximately. One can respectively locate the two cell centers that bound these velocities, and then use a linear interpolation to evaluate the needed numerical flux at these new wave numbers. The case of $\xi_{k}<0$ is treated similarly. All above can be done symmetrically for the horizontal interface.

We explain how to evaluate $a_{i+\frac{1}{2}, j k l}^{\tau \pm}(\tau=p, s)$ in the following algorithm and $a_{i, j+\frac{1}{2}, k l}^{\tau \pm}$ $(\tau=p, s)$ can be obtained similarly.

\section{Algorithm :}


- if $\xi_{k}>0$

$$
a_{i+\frac{1}{2}, j k l}^{p-}=a_{i j k l}^{p}, a_{i+\frac{1}{2}, j k l}^{s-}=a_{i j k l}^{s}, \xi_{k_{1}}=-\xi_{k}
$$

To get $a_{i+\frac{1}{2}, j k l}^{p+}$, we first need to get three new wave numbers $\xi^{p p-}, \xi^{s p-}, \xi^{s p+}$ as following:

$\diamond$ Transmission from a $\mathrm{P}$-wave to the $\mathrm{P}$-wave :

$$
\begin{aligned}
& \text { if }\left(\frac{C_{i+\frac{1}{2}, j}^{p+}}{C_{i+\frac{1}{2}, j}^{p}}\right)^{2}\left(\xi_{k}\right)^{2}+\left[\left(\frac{C_{i+\frac{1}{2}, j}^{p+}}{C_{i+\frac{1}{2}, j}^{p-}}\right)^{2}-1\right]\left(\eta_{l}\right)^{2}>0 \\
& \xi^{p p-}=\sqrt{\left(\frac{C_{i+\frac{1}{2}, j}^{p+}}{C_{i+\frac{1}{2}, j}^{p}}\right)^{2}\left(\xi_{k}\right)^{2}+\left[\left(\frac{C_{i+\frac{1}{2}, j}^{p+}}{C_{i+\frac{1}{2}, j}^{p-j}}\right)^{2}-1\right]\left(\eta_{l}\right)^{2},} \\
& \xi_{m_{p}} \leq \xi^{p p-}<\xi_{m_{p}+1} \text { for some integer } m_{p},
\end{aligned}
$$

else

$$
\text { set } m_{p}=1 \text {. }
$$

end

$\diamond$ Transmission from a S-wave to the $\mathrm{P}$-wave :

$$
\begin{aligned}
& \text { if }\left(\frac{C_{i+\frac{1}{2}, j}^{p+}}{C_{i+\frac{1}{2}, j}^{s-j}}\right)^{2}\left(\xi_{k}\right)^{2}+\left[\left(\frac{C_{i+\frac{1}{2}, j}^{p+}}{C_{i+\frac{1}{2}, j}^{s-j}}\right)^{2}-1\right]\left(\eta_{l}\right)^{2}>0 \\
& \xi^{s p-}=\sqrt{\left(\frac{C_{i+\frac{1}{2}, j}^{p+}}{C_{i+\frac{1}{2}, j}^{s-j}}\right)^{2}\left(\xi_{k}\right)^{2}+\left[\left(\frac{C_{i+\frac{1}{2}, j}^{p+}}{C_{i+\frac{1}{2}, j}^{s-j}}\right)^{2}-1\right]\left(\eta_{l}\right)^{2},} \\
& \xi_{n_{p}} \leq \xi^{s p-}<\xi_{n_{p}+1} \text { for some integer } n_{p},
\end{aligned}
$$

else

$$
\text { set } n_{p}=1 \text {. }
$$

end

$\diamond$ Reflection of the $\mathrm{P}$-wave from a S-wave :

$$
\begin{aligned}
& \text { if }\left(\frac{C_{i+\frac{1}{2}, j}^{p+}}{C_{i+\frac{1}{2}, j}^{s+}}\right)^{2}\left(\xi_{k}\right)^{2}+\left[\left(\frac{C_{i+\frac{1}{2}, j}^{p+}}{C_{i+\frac{1}{2}, j}^{s+1}}\right)^{2}-1\right]\left(\eta_{l}\right)^{2}>0 \\
& \xi^{s p+}=-\sqrt{\left(\frac{C_{i+\frac{1}{2}, j}^{p+}}{C_{i+\frac{1}{2}, j}^{s+j}}\right)^{2}\left(\xi_{k}\right)^{2}+\left[\left(\frac{C_{i+\frac{1}{2}, j}^{p+}}{C_{i+\frac{1}{2}, j}^{s}}\right)^{2}-1\right]\left(\eta_{l}\right)^{2},} \\
& \xi_{r_{p}} \leq \xi^{s p+}<\xi_{r_{p}+1} \text { for some integer } r_{p},
\end{aligned}
$$


else

$$
\text { set } r_{p}=1 \text {. }
$$

end

Then

$$
\begin{aligned}
a_{i+\frac{1}{2}, j k l}^{p+}= & \beta_{T}^{P P}\left(\frac{\xi_{m_{p}+1}-\xi^{p p-}}{\Delta \xi} a_{i j, m_{p}, l}^{p}+\frac{\xi^{p p-}-\xi_{m_{p}}}{\Delta \xi} a_{i j, m_{p}+1, l}^{p}\right) \\
& +\beta_{R}^{P P} a_{i+1, j, k_{1}, l}^{p} \\
& +\beta_{T}^{S P}\left(\frac{\xi_{n_{p}+1}-\xi^{s p-}}{\Delta \xi} a_{i j, n_{p}, l}^{s}+\frac{\xi^{s p-}-\xi_{n_{p}}}{\Delta \xi} a_{i j, n_{p}+1, l}^{s}\right) \\
& +\beta_{R}^{S P}\left(\frac{\xi_{r_{p}+1}-\xi^{s p+}}{\Delta \xi} a_{i+1, j, r_{p}, l}^{s}+\frac{\xi^{s p+}-\xi_{r_{p}}}{\Delta \xi} a_{i+1, j, r_{p}+1, l}^{s}\right)
\end{aligned}
$$

where the energy coefficients $\beta_{T}^{P P}, \beta_{R}^{P P}, \beta_{T}^{S P}, \beta_{R}^{S P}$ are obtained as shown in the matrix (2.12) for $\left(\xi_{k}, \eta_{l}\right)$.

Remark 1 As mentioned earlier, when the wave number becomes imaginary, we just set its corresponding energy coefficient to be zero. Hence we can arbitrarily pick a integer value for $m_{p}, n_{p}, r_{p}$. For simplicity, we set them to be one if their corresponding wave numbers are imaginary. It is similar in the construction of flux for $S$-wave in the following.

To get $a_{i+\frac{1}{2}, j k l}^{s+}$, we also need to get three new wave numbers $\xi^{p s-}, \xi^{s s-}, \xi^{p s+}$ as following:

$\diamond$ Transmission from a $\mathrm{P}$-wave to the $\mathrm{S}$-wave :

$$
\begin{aligned}
& \text { if }\left(\frac{C_{i+\frac{1}{2}, j}^{s+}}{C_{i+\frac{1}{2}, j}^{p-}}\right)^{2}\left(\xi_{k}\right)^{2}+\left[\left(\frac{C_{i+\frac{1}{2}, j}^{s+}}{C_{i+\frac{1}{2}, j}^{p-}}\right)^{2}-1\right]\left(\eta_{l}\right)^{2}>0 \\
& \xi^{p s-}=\sqrt{\left(\frac{C_{i+\frac{1}{2}, j}^{s+}}{C_{i+\frac{1}{2}, j}^{p-}}\right)^{2}\left(\xi_{k}\right)^{2}+\left[\left(\frac{C_{i+\frac{1}{2}, j}^{s+}}{C_{i+\frac{1}{2}, j}^{p-}}\right)^{2}-1\right]\left(\eta_{l}\right)^{2},} \\
& \quad \xi_{m_{s}} \leq \xi^{p s-}<\xi_{m_{s}+1} \text { for some integer } m_{s}, \\
& \text { else } \\
& \quad \text { set } m_{s}=1 .
\end{aligned}
$$

end

$\diamond$ Transmission from a S-wave to the S-wave :

$$
\text { if }\left(\frac{C_{i+\frac{1}{2}, j}^{s+}}{C_{i+\frac{1}{2}, j}^{s-}}\right)^{2}\left(\xi_{k}\right)^{2}+\left[\left(\frac{C_{i+\frac{1}{2}, j}^{s+}}{C_{i+\frac{1}{2}, j}^{s-}}\right)^{2}-1\right]\left(\eta_{l}\right)^{2}>0
$$


$\xi^{s s-}=\sqrt{\left(\frac{C_{i+\frac{1}{2}, j}^{s+}}{C_{i+\frac{1}{2}, j}^{s-}}\right)^{2}\left(\xi_{k}\right)^{2}+\left[\left(\frac{C_{i+\frac{1}{2}, j}^{s+}}{C_{i+\frac{1}{2}, j}^{s-}}\right)^{2}-1\right]\left(\eta_{l}\right)^{2}}$,

$\xi_{n_{s}} \leq \xi^{s s-}<\xi_{n_{s}+1}$ for some integer $n_{s}$,

else

set $n_{s}=1$.

end

$\diamond$ Reflection of the S-wave from a P-wave :

if $\left(\frac{C_{i+\frac{1}{2}, j}^{s+}}{C_{i+\frac{1}{2}, j}^{p+}}\right)^{2}\left(\xi_{k}\right)^{2}+\left[\left(\frac{C_{i+\frac{1}{2}, j}^{s+}}{C_{i+\frac{1}{2}, j}^{p+}}\right)^{2}-1\right]\left(\eta_{l}\right)^{2}>0$

$\xi^{p s+}=-\sqrt{\left(\frac{C_{i+\frac{1}{2}, j}^{s+}}{C_{i+\frac{1}{2}, j}^{p+}}\right)^{2}\left(\xi_{k}\right)^{2}+\left[\left(\frac{C_{i+\frac{1}{2}, j}^{s+}}{C_{i+\frac{1}{2}, j}^{p+}}\right)^{2}-1\right]\left(\eta_{l}\right)^{2}}$,

$\xi_{r_{s}} \leq \xi^{p s+}<\xi_{r_{s}+1}$ for some integer $r_{s}$

else

set $r_{s}=1$.

end

Then

$$
\begin{aligned}
a_{i+\frac{1}{2}, j k l}^{s+}= & \beta_{T}^{P S}\left(\frac{\xi_{m_{s}+1}-\xi^{p s-}}{\Delta \xi} a_{i j, m_{s}, l}^{p}+\frac{\xi^{p s-}-\xi_{m_{s}}}{\Delta \xi} a_{i j, m_{s}+1, l}^{p}\right) \\
& +\beta_{R}^{S S} a_{i+1, j, k_{1}, l}^{s}{ }^{s s-} \\
& +\beta_{T}^{S S}\left(\frac{\xi_{n_{s}+1}-\xi^{s s}}{\Delta \xi} a_{i j, n_{s}, l}^{s}+\frac{\xi^{s s-}-\xi_{n_{s}}}{\Delta \xi} a_{i j, n_{s}+1, l}^{s}\right) \\
& +\beta_{R}^{P S}\left(\frac{\xi_{r_{s}+1}-\xi^{p s+}}{\Delta \xi} a_{i+1, j, r_{s}, l}^{p}+\frac{\xi^{p s+}-\xi_{r_{s}}}{\Delta \xi} a_{i+1, j, r_{s}+1, l}^{p}\right)
\end{aligned}
$$

where the energy coefficients $\beta_{T}^{P S}, \beta_{R}^{S S}, \beta_{T}^{S S}, \beta_{R}^{P S}$ are obtained as shown in the matrix (2.12) for $\left(\xi_{k}, \eta_{l}\right)$.

- if $\xi_{k}<0$

$$
a_{i+\frac{1}{2}, j k l}^{p+}=a_{i+1, j k l}^{p}, a_{i+\frac{1}{2}, j k l}^{s+}=a_{i+1, j k l}^{s},
$$

$a_{i+\frac{1}{2}, j k l}^{p-}$ and $a_{i+\frac{1}{2}, j k l}^{s-}$ can be obtained similarly by the above way.

The above algorithm for evaluating numerical fluxes is of first order. One can obtain a second order flux by incorporating a slope limiter, such as the van Leer or 
minmod slope limiter [16], into the above algorithm. This can be achieved by replacing $f_{i j k l}$ with $f_{i j k l}+\frac{\Delta x}{2} s_{i j k l}$, and replacing $f_{i+1, j k l}$ with $f_{i+1, j k l}-\frac{\Delta x}{2} s_{i+1, j k l}$ in the above algorithm, where $s_{i j k l}$ is the slope limiter in the $x$-direction. It is similar in the $y$ direction, $\xi$-direction and $\eta$-direction. For the numerical examples in the next section, we just use the conventional van Leer slope limiter which works well for our examples. It is mainly because the slope along the interface is just zero in those examples. In general, one probably needs to modify slope limiter near the interface, again by using the Hamiltonian preserving principle across the interface, as in [13].

After the spatial discretization is specified, one can use any time discretization for the time derivative.

\subsection{Positivity and $l^{\infty}$ contraction}

It is important for the numerical scheme to guarantee the positivity of $a^{p}$ and $a^{s}$ when solving Liouville equations (4.1) and (4.2) because they represent the phase energy density.

We first consider the scheme on (4.1) using the first order numerical flux, and the forward Euler method in time. We consider the case when there are both a vertical and a horizontal interface in $x-y$ plane. Assume that $\xi_{k}>0, \eta_{l}>0$ and $c_{i+\frac{1}{2}, j}^{p-}<c_{i-\frac{1}{2}, j}^{p+}$, $c_{i, j+\frac{1}{2}}^{p-}<c_{i, j-\frac{1}{2}}^{p+}$ for all $i$ and $j$ (the other cases will similarly result in the same conclusion). The scheme becomes

$$
\begin{aligned}
\frac{a_{i j k l}^{p(n+1)}-a_{i j k l}^{p(n)}}{\Delta t} & +\frac{c_{i j}^{p} \xi_{k}}{\sqrt{\xi_{k}^{2}+\eta_{l}^{2}}} \frac{a_{i j k l}^{p}-a_{i-\frac{1}{2}, j k l}^{p+}}{\Delta x} \\
& +\frac{c_{i j}^{p} \eta_{l}}{\sqrt{\xi_{k}^{2}+\eta_{l}^{2}}} \frac{a_{i j k l}^{p}-a_{i, j-\frac{1}{2}, k l}^{p+}}{\Delta y} \\
& -\frac{c_{i+\frac{1}{2}, j}^{p-}-c_{i-\frac{1}{2}, j}^{p+}}{\Delta x \Delta \xi} \sqrt{\xi_{k}^{2}+\eta_{l}^{2}}\left(a_{i j k l}^{p}-a_{i j, k-1, l}^{p}\right) \\
& -\frac{c_{i, j+\frac{1}{2}}^{p-}-c_{i, j-\frac{1}{2}}^{p+}}{\Delta y \Delta \eta} \sqrt{\xi_{k}^{2}+\eta_{l}^{2}}\left(a_{i j k l}^{p}-a_{i j k, l-1}^{p}\right)=0
\end{aligned}
$$

with

$$
\begin{aligned}
a_{i-\frac{1}{2}, j k l}^{p+}= & d_{t_{1}}^{p} a_{i-1, j, m_{p}, l}^{p}+d_{t_{2}}^{p} a_{i-1, j, m_{p}+1, l}^{p}+d_{r}^{p} a_{i j, k_{1}, l}^{p} \\
+ & d_{t_{1}}^{s} a_{i-1, j, n_{p}, l}^{s}+d_{t_{2}}^{s} a_{i-1, j, n_{p}+1, l}^{s}+d_{r_{1}}^{s} a_{i j, r_{p}, l}^{s}+d_{r_{2}}^{s} a_{i j, r_{p}+1, l}^{s}
\end{aligned}
$$

where $d_{t_{1}}^{p}, d_{t_{2}}^{p}, d_{r}^{p}, d_{t_{1}}^{s}, d_{t_{2}}^{s}, d_{r_{1}}^{s}, d_{r_{2}}^{s}$ are non-negative and $d_{t_{1}}^{p}+d_{t_{2}}^{p}+d_{r}^{p}+d_{t_{1}}^{s}+d_{t_{2}}^{s}+d_{r_{1}}^{s}+d_{r_{2}}^{s}=$ 1. It is similar for $a_{i, j-\frac{1}{2}, k l}^{p+}$. We omit the superscript $n$ of $a^{p}$. The above scheme can be 
rewritten as

$$
\begin{aligned}
& a_{i j k l}^{p(n+1)}=\left(1-\frac{c_{i j}^{p} \xi_{k}}{\sqrt{\xi_{k}^{2}+\eta_{l}^{2}}} \frac{\Delta t}{\Delta x}-\frac{c_{i j}^{p} \eta_{l}}{\sqrt{\xi_{k}^{2}+\eta_{l}^{2}}} \frac{\Delta t}{\Delta y}\right. \\
& -\frac{\left|c_{i+\frac{1}{2}, j}^{p-}-c_{i-\frac{1}{2}, j}^{p+}\right|}{\Delta x} \sqrt{\xi_{k}^{2}+\eta_{l}^{2}} \frac{\Delta t}{\Delta \xi} \\
& \left.-\frac{\left|c_{i, j+\frac{1}{2}}^{p-}-c_{i, j-\frac{1}{2}}^{p+}\right|}{\Delta y} \sqrt{\xi_{k}^{2}+\eta_{l}^{2}} \frac{\Delta t}{\Delta \eta}\right) \cdot a_{i j k l}^{p} \\
& +\frac{c_{i j}^{p} \xi_{k}}{\sqrt{\xi_{k}^{2}+\eta_{l}^{2}}} \frac{\Delta t}{\Delta x} \cdot a_{i-\frac{1}{2}, j k l}^{p+}+\frac{c_{i j}^{p} \eta_{l}}{\sqrt{\xi_{k}^{2}+\eta_{l}^{2}}} \frac{\Delta t}{\Delta y} \cdot a_{i, j-\frac{1}{2}, k l}^{p+} \\
& +\frac{\left|c_{i+\frac{1}{2}, j}^{p-}-c_{i-\frac{1}{2}, j}^{p+}\right|}{\Delta x} \sqrt{\xi_{k}^{2}+\eta_{l}^{2}} \frac{\Delta t}{\Delta \xi} \cdot a_{i j, k-1, l}^{p} \\
& +\frac{\left|c_{i, j+\frac{1}{2}}^{p-}-c_{i, j-\frac{1}{2}}^{p+}\right|}{\Delta y} \sqrt{\xi_{k}^{2}+\eta_{l}^{2}} \frac{\Delta t}{\Delta \eta} \cdot a_{i j k, l-1}^{p}
\end{aligned}
$$

Similarly, we can have the same discretization for $a^{s}$ in S-wave. To investigate the positivity of scheme (4.7), we just need to prove if $a_{i j k l}^{p(n)} \geq 0$ and $a_{i j k l}^{s(n)} \geq 0$ for all $(i, j, k, l)$, then this is also true for $a_{i j k l}^{p(n+1)} \geq 0$ and $a_{i j k l}^{s(n+1)} \geq 0$ for all $(i, j, k, l)$. Clearly one just needs to show that all the coefficients before $a^{p(n)}$ are non-negative. A sufficient condition for this is

$$
\begin{aligned}
1 & -\frac{c_{i j}^{p} \xi_{k}}{\sqrt{\xi_{k}^{2}+\eta_{l}^{2}}} \frac{\Delta t}{\Delta x}-\frac{c_{i j}^{p} \eta_{l}}{\sqrt{\xi_{k}^{2}+\eta_{l}^{2}}} \frac{\Delta t}{\Delta y} \\
& -\frac{\left|c_{i+\frac{1}{2}, j}^{p-}-c_{i-\frac{1}{2}, j}^{p+}\right|}{\Delta x} \sqrt{\xi_{k}^{2}+\eta_{l}^{2}} \frac{\Delta t}{\Delta \xi}-\frac{\left|c_{i, j+\frac{1}{2}}^{p-}-c_{i, j-\frac{1}{2}}^{p+}\right|}{\Delta y} \sqrt{\xi_{k}^{2}+\eta_{l}^{2}} \frac{\Delta t}{\Delta \eta} \geq 0
\end{aligned}
$$

or sufficiently,

$$
\Delta t \max _{i, j, k, l}\left[c_{i j}^{p} \sqrt{\frac{1}{\Delta x^{2}}+\frac{1}{\Delta y^{2}}}+\frac{\frac{\left|c_{i+\frac{1}{2}, j}^{p-}-c_{i-\frac{1}{2}, j}^{p+}\right|}{\Delta x} \sqrt{\xi_{k}^{2}+\eta_{l}^{2}}}{\Delta \xi}+\frac{\frac{\left|c_{i, j+\frac{1}{2}}^{p-}-c_{i, j-\frac{1}{2}}^{p+}\right|}{\Delta y} \sqrt{\xi_{k}^{2}+\eta_{l}^{2}}}{\Delta \eta}\right] \leq 1
$$

which is similar for S-wave.

The quantity $\frac{\left|c_{i+\frac{1}{2}, j}^{p-}-c_{i-\frac{1}{2}, j}^{p+}\right|}{\Delta x}$ and $\frac{\left|c_{i, j+\frac{1}{2}}^{p-}-c_{i, j-\frac{1}{2}}^{p+}\right|}{\Delta y}$ now represent the wave speed gradient at the smooth points, which have finite upper bounds since $c^{p}$ has a bounded variation. So our scheme allows a time step $\Delta t=O(|\Delta \mathbf{x}|,|\Delta \mathbf{k}|)$.

According to the study in [21], our second order scheme is positive under the half CFL condition which says the constant on the right hand side of (4.8) is $1 / 2$. 
The $l^{\infty}$-contracting property of this scheme follows easily, since under (4.8), all the coefficients in (4.7) are positive and the sum of them is 1. Thus

$$
\max \left(\left\|a^{p(n)}\right\|_{\infty},\left\|a^{s(n)}\right\|_{\infty}\right) \leq \max \left(\left\|a^{p(0)}\right\|_{\infty},\left\|a^{s(0)}\right\|_{\infty}\right) .
$$

\section{Curved Interface}

So far we only described the algorithm for flat interface aligned with the meshes. In this section we extend it to the case of curved interfaces. The idea is to use the Hamiltonian preservation princinple locally in the direction normal to the interface.

Suppose we have an interface in the solid media given by a curve $\mathcal{C}$, defined by the equation

$$
\mathcal{F}(x, y)=0 .
$$

We classify the grid points into the following categories (see Figure 2):

- Sweep along the horizontal direction for each $j$ and flag the points adjacent to the interface from the right and the left. These points are called horizontal irregular points and labelled with 'H'.

- Sweep along the vertical direction for each $i$ and flag the points which are adjacent to the interface from the top and the bottom. These points are called vertical irregular points and labelled with ' $\mathrm{V}$ '.

- All the other points are regular points.

After we determine the irregular points, the cell interface between two horizontally adjacent irregular points and also two vertically adjacent irregular points can be seen as an approximation to the real interface curve $\mathcal{C}$. By doing this, we can still assign our averaged wave speed as what we introduce in Section 4.2 .

One more important issue here is to choose the correct direction for reflection and transmission of incident ray so that we can find the appropriate splitting flux $a_{i+\frac{1}{2}, j k l}^{\tau-}$, $a_{i-\frac{1}{2}, j k l}^{\tau+}, a_{i, j+\frac{1}{2}, k l}^{\tau-}, a_{i, j-\frac{1}{2}, k l}^{\tau+}$ at the interface. To explain how to deal with that, now take one pair of horizontal irregular points $\left(x_{i}, y_{j+1}\right),\left(x_{i+1}, y_{j+1}\right)$ in Figure 2 as a demo :

Connecting these two points by a dashed line as in the figure and it must have an intersection with the interface, say $P$. Setting up a local coordinate system $\left(x^{\prime}, y^{\prime}\right)$ at $P$ , in which $x^{\prime}$ is normal to the interface $\mathcal{C}$, and $y^{\prime}$ is tangential to $\mathcal{C}$, at the point $P$. So the original particle velocity $\mathbf{k}=(\xi, \eta)$ will become $\mathbf{k}^{\prime}=\left(\xi^{\prime}, \eta^{\prime}\right)$ in this new coordinate system with $\mathbf{k}^{\prime}=Q \mathbf{k}$ where the rotation matrix $Q$ is given by

$$
Q=\left(\begin{array}{cc}
\cos \theta & \sin \theta \\
-\sin \theta & \cos \theta
\end{array}\right)
$$

Here, $\theta$ is the rotation angle, i.e. the angle between the $x$-axis and $x^{\prime}$-axis. Then we can get the transmission and reflection coefficients $\beta_{R}^{P P}, \beta_{T}^{P S}, \beta_{R}^{P S}$, etc. for the incident 


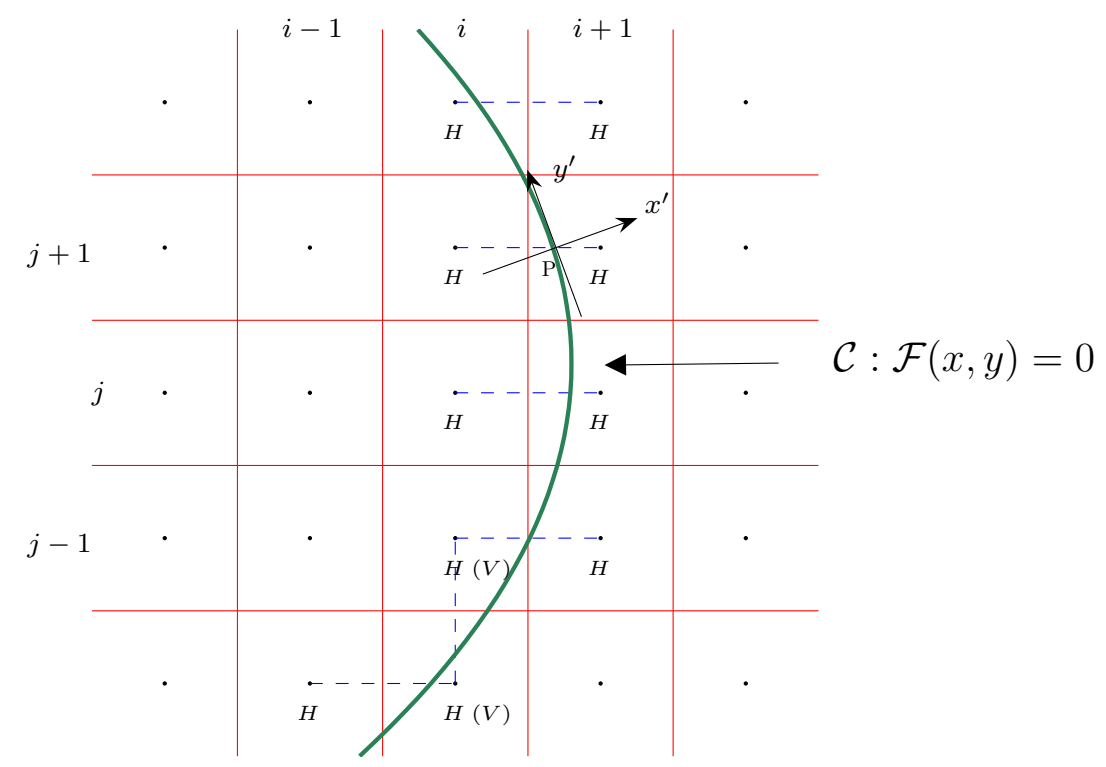

Figure 2: Two dimensional interface with irregular points labelled as ' $\mathrm{H}$ ' and ' $\mathrm{V}$ '. The coordinates $x^{\prime}$ and $y^{\prime}$ are defined locally at the point $P$ on the interface.

ray at the point $P$ in this local coordinate system by the way introduced before, with axis $y^{\prime}$ as the interface. And for the corresponding wave numbers $\xi^{p p-}, \xi^{s p-}$, etc. of the transmitted and reflected rays, it can be obtained by applying the Hamiltonian preserving principle for $\mathbf{k}^{\prime}$ in the local coordinate system and then transforming them back to the original system by multiplying $Q^{-1}$. The new wave numbers obtained in this way still have a constant Hamiltonian in the original coordinate system because $Q$ is an orthogonal matrix and $\left|\mathbf{k}^{\prime}\right|=|\mathbf{k}|$.

After getting the coefficients and corresponding wave numbers, we can calculate the splitting wave fluxes $f_{i+1 / 2, j}^{ \pm}$and $f_{i, j+1 / 2}^{ \pm}$by the Algorithm in Section 4. Here effectively we moved $\mathrm{P}$ to the nearest mesh point, resulting an $O(\Delta x, \Delta y)$ error. One can improve the accuracy here using subcell, namely, adding a grid point at $P$. This can be done in a standard way, but will not be explored in this paper.

The above techniques can be applied for any interface curve as long as there is an explicit expression for it. Of course, it can be applied for the cases like Example 6.1 and Example 6.2 although it's not necessary. We use it in Example 6.3 and the interface in that case can be captured sharply, which is shown in the end. Also, positivity and $l^{\infty}$ contraction of the scheme are not effected in this curved interface situation because the basic algorithm is still the same as before. 


\section{$6 \quad$ Numerical examples}

We consider the high frequency limit solution of the elastic wave in 2D plane consisting of two different kinds of solids in welded contact, with the initial data

$$
\mathbf{u}_{0}=\mathbf{A}_{0} \exp \left(i \frac{S_{0}}{\epsilon}\right)
$$

Then as $\epsilon \rightarrow 0$, this limit will be the solution of (4.1) and (4.2) with the initial condition (4.3) and (4.4).

For arbitrarily given $\mathbf{A}_{0}$ and $\nabla S_{0}$ in (6.1), we need to decompose $\mathbf{A}_{0}$ into two components: one is parallel to the direction of $\nabla S_{0}$, which is the amplitude of $\mathrm{P}$-wave and denoted as $\mathbf{A}_{0}^{p}$; the other one is normal to the direction of $\nabla S_{0}$, which is the amplitude of S-wave and denoted as $\mathbf{A}_{0}^{s}$. Therefore,

$$
\left|\mathbf{A}_{0}^{p}\right|=\frac{\mathbf{A}_{0} \cdot \nabla S_{0}}{\left|\nabla S_{0}\right|}, \quad\left|\mathbf{A}_{0}^{s}\right|=\sqrt{\left|\mathbf{A}_{0}\right|^{2}-\left|\mathbf{A}_{0}^{p}\right|^{2}}
$$

Then $\mathcal{E}_{0}^{p}(\mathbf{x})$ and $\mathcal{E}_{0}^{s}(\mathbf{x})$ in the initial data (4.3) and (4.4) is given accordingly as:

$$
\mathcal{E}_{0}^{p}(\mathbf{x})=\frac{\left|\mathbf{A}_{0}^{p}\right|^{2}}{\left|c^{p}\right|^{2}}, \quad \mathcal{E}_{0}^{s}(\mathbf{x})=\frac{\left|\mathbf{A}_{0}^{s}\right|^{2}}{\left|c^{s}\right|^{2}}
$$

In the following numerical examples, we use (6.2) to assign the initial data for the Liouville equations (4.1) and (4.2). For numerical computation, we use the first order Forward Euler for the time discretization and the second order flux with the van Leer slope limiter for the spatial discretization.

We are interested in computing the energy density $E(t, \mathbf{x})$ which is given by

$$
\begin{aligned}
E(t, \mathbf{x}) & =\int a^{p}(t, \mathbf{x}, \mathbf{k}) d \mathbf{k}+\int a^{s}(t, \mathbf{x}, \mathbf{k}) d \mathbf{k} \\
& =\mathcal{E}^{p}(t, \mathbf{x})+\mathcal{E}^{s}(t, \mathbf{x})
\end{aligned}
$$

Example 6.1 The interface of two solids is located at $x=0$. The solid on the left half plane $x<0$ has the density $\rho_{1}=1$ and Lamé constants $\lambda_{1}=0.2, \mu_{1}=0.5$; and the solid on the right half plane $x>0$ has the density $\rho_{2}=0.8$ and Lamé constants $\lambda_{2}=0.4, \mu_{2}=0.8$. So it results in the discontinuous wave speeds as

$$
c^{p}(x, y)=\left\{\begin{array}{lll}
\sqrt{1.2} & \text { if } & x<0 \\
\sqrt{2.5} & \text { if } & x>0
\end{array}\right.
$$

and

$$
c^{s}(x, y)=\left\{\begin{array}{lll}
\sqrt{0.5} & \text { if } \quad x<0 \\
1 & \text { if } \quad x>0 .
\end{array}\right.
$$

The initial conditions are given by $\mathbf{A}_{0}=(1,0.5)$ and $\nabla S_{0}=(0.1,0.2)$. So the corresponding $\mathcal{E}_{0}^{p}(\mathbf{x})$ and $\mathcal{E}_{0}^{s}(\mathbf{x})$ are

$$
\mathcal{E}_{0}^{p}(x, y)=\left\{\begin{array}{lll}
\frac{4}{6} & \text { if } & x<0 \\
\frac{{ }_{4}}{12.5} & \text { if } & x>0
\end{array}\right.
$$


and

$$
\mathcal{E}_{0}^{s}(x, y)=\left\{\begin{array}{lll}
\frac{9}{10} & \text { if } & x<0 \\
\frac{9}{20} & \text { if } & x>0
\end{array}\right.
$$

The exact solutions of $\mathcal{E}^{p}(t, \mathbf{x})$ and $\mathcal{E}^{s}(t, \mathbf{x})$ are given by

$$
\mathcal{E}^{p}(t, x, y)=\left\{\begin{array}{lll}
\frac{4}{12.5} & \text { if } & x>\sqrt{0.5} t \\
0 & \text { if } & 0<x<\sqrt{0.5} t \\
\frac{4}{6} \beta_{R}^{P P}+\frac{4}{6} & \text { if } & -\sqrt{\frac{1.2}{5}} t<x<0 \\
\frac{4}{6} & \text { if } & x<-\sqrt{\frac{1.2}{5}} t
\end{array}\right.
$$

and

$$
\mathcal{E}^{s}(t, x, y)=\left\{\begin{array}{lll}
\frac{9}{20} & \text { if } & x>\sqrt{\frac{1}{3}} t \\
\frac{9}{20}+\frac{0.8}{\sqrt{2}} \beta_{T}^{P S} & \text { if } & \sqrt{\frac{1}{5}} t<x<\sqrt{\frac{1}{3}} t \\
\frac{0.8}{\sqrt{2}} \beta_{T}^{P S} & \text { if } & 0<x<\sqrt{\frac{1}{5}} t \\
\frac{9}{10}+\frac{9}{10} \beta_{R}^{S S}+\frac{4}{5 \sqrt{2}} \beta_{R}^{P S} & \text { if } & -\sqrt{0.1} t<x<0 \\
\frac{9}{10}+\frac{4}{5 \sqrt{2}} \beta_{R}^{P S} & \text { if } & -\sqrt{\frac{1}{3}} t<x<-\sqrt{0.1} t \\
\frac{9}{10} & \text { if } & x<-\sqrt{\frac{1}{3}} t
\end{array}\right.
$$

where the physically relevant values for the reflection and transmission coefficients at the interface are $\beta_{R}^{P P}=0.7271, \beta_{R}^{P S}=0.1041, \beta_{T}^{P S}=0.1688, \beta_{R}^{S S}=1$.

In the computation, we approximate the $2 \mathrm{D} \boldsymbol{\delta}$-function initial data (4.3) and (4.4) by the product of a discrete delta function in $1 \mathrm{D}[6]$ :

$$
\delta_{h}(x)=\left\{\begin{array}{cl}
\frac{1}{h}\left(1-\frac{|x|}{h}\right), & |x| \leq h \\
0, & \text { otherwise }
\end{array}\right.
$$

with $h=\Delta \xi=\Delta \eta$ for regularization.

We choose the time step as $\Delta t=\frac{1}{2} \Delta x$. The computational domain is chosen as $(x, y, \xi, \eta) \in[-0.2,0.2] \times[-0.1,0.1] \times[-0.5,0.5] \times[0.1,0.3]$. Since the interface is parallel to the $\mathrm{y}$-axis, the material is heterogeneous along the $\mathrm{x}$-direction and it is homogeneous along the y-direction. Hence we just need few grid points in the $y$ and $\eta$ directions and put more grid points in the $x$ and $\xi$ directions. Table 1 presents the $l^{1}$ errors, at $t=0.25$, of energy density $\mathcal{E}^{p}$ and $\mathcal{E}^{s}$ computed with different meshes $M_{1} \times M_{2} \times N_{1} \times N_{2}$ where we fix $M_{2}=10, N_{1}=80, N_{2}=20$ and $M_{1}$ is increased from 101 to 801 . The convergence rate is higher at the beginning and decreasing with time going on. So the average convergence rate is around 0.96 for $\mathrm{P}$-wave and 0.76 for $\mathrm{S}$-wave. The rate is around 0.8 for the total energy density.

Figure 3 shows the energy density $\mathcal{E}^{p}$ and $\mathcal{E}^{s}$ at $t=0.25$ along with the exact solution. Figure 4 and 5 present some cross sections which compare the numerical solution with the exact solution at different time $t=0.1, t=0.18, t=0.25, t=0.30$ respectively. The numerical method gives a sharp material interface at $x=0$, and high resolution in other propagating discontinuities. 


\begin{tabular}{ccccc}
\hline grid points $\left(M_{1}\right)$ & 101 & 201 & 401 & 801 \\
\hline $\mathcal{E}^{p}$ & $0.29686 \mathrm{E}-3$ & $0.15115 \mathrm{E}-3$ & $0.08558 \mathrm{E}-3$ & $0.04483 \mathrm{E}-3$ \\
$\mathcal{E}^{s}$ & $0.88143 \mathrm{E}-3$ & $0.47732 \mathrm{E}-3$ & $0.30472 \mathrm{E}-3$ & $0.20577 \mathrm{E}-3$ \\
$E=\mathcal{E}^{p}+\mathcal{E}^{s}$ & $1.17518 \mathrm{E}-3$ & $0.62762 \mathrm{E}-3$ & $0.38986 \mathrm{E}-3$ & $0.25017 \mathrm{E}-3$ \\
\hline
\end{tabular}

Table 1: Example 6.1. $l^{1}$ error of energy density $\mathcal{E}^{p}, \mathcal{E}^{s}$ and the total energy density $E(t, \mathbf{x})$ using different meshes at $t=0.25$.

Example 6.2 The interfaces of two solids are located at the positive $x$-axis and the positive $y$-axis. The solid in the first quadrant $(x>0, y>0)$ has density $\rho_{1}=0.8$, Lamé constants $\lambda_{1}=0.4, \mu_{1}=0.8$; and the solid elsewhere has density $\rho_{2}=1$, Lamé constants $\lambda_{2}=0.2, \mu_{2}=0.3$. So it results in the discontinuous wave speeds as

$$
c^{p}(x, y)= \begin{cases}\sqrt{2.5} & \text { if } x>0, y>0 \\ \sqrt{0.8} & \text { otherwise }\end{cases}
$$

and

$$
c^{s}(x, y)= \begin{cases}1 & \text { if } x>0, y>0 \\ \sqrt{0.3} & \text { otherwise. }\end{cases}
$$

The initial conditions are given by

$$
\mathbf{A}_{0}= \begin{cases}(0,0) & \text { if } x>-0.1, y>-0.1 \\ (1,2) & \text { otherwise }\end{cases}
$$

and $\nabla S_{0}=(0.1,0.1)$. So the corresponding $\mathcal{E}_{0}^{p}(\mathbf{x})$ and $\mathcal{E}_{0}^{s}(\mathbf{x})$ are

$$
\mathcal{E}_{0}^{p}(x, y)= \begin{cases}0 & \text { if } x>-0.1, y>-0.1 \\ \frac{9}{1.6} & \text { otherwise }\end{cases}
$$

and

$$
\mathcal{E}_{0}^{s}(x, y)= \begin{cases}0 & \text { if } x>-0.1, y>-0.1 \\ \frac{1}{0.6} & \text { otherwise }\end{cases}
$$

For exact solution, the reflection and transmission coefficients at the interface are $\beta_{R}^{P P}=0.3953, \beta_{R}^{P S}=0.2347, \beta_{T}^{P S}=0.3699, \beta_{R}^{S S}=1$. The graphs of exact solutions at different times are shown in the left columns of Figure 6 and Figure 7.

We here use the same discretization for $\boldsymbol{\delta}$-function as in the first example and choose the time step as $\Delta t=\frac{1}{8} \Delta x$. And the computational domain is chosen as $(x, y, \xi, \eta) \in$ $[-0.2,0.2] \times[-0.2,0.2] \times[-0.4,0.4] \times[-0.4,0.4]$. In this example, the material has the interfaces both in $\mathrm{x}$-direction and $\mathrm{y}$-direction. So we should treat these two directions symmetrically. The right columns of Figure 6 and Figure 7 present the numerical energy density for $\mathrm{P}$-wave and S-wave respectively, which can be compared with the corresponding exact solution on the left column of the graphs. Figure 8 and 9 display the sectional view along the line $y=0.08$ for energy density of the $\mathrm{P}$-wave and the 
S-wave at four different times $t=0.1, t=0.18, t=0.23, t=0.45$, which shows the numerical solutions agree well with the exact solutions. The material interfaces are captured sharply.

Table 2 presents the $l^{1}$ error, at $t=0.5$, of energy density for P-wave and S-wave respectively and also of total energy density $E(t, \mathbf{x})$ computed with the different meshes $M_{1} \times M_{2} \times N_{1} \times N_{2}$ where we fix $N_{1} \times N_{2}=80 \times 80$ and $M_{1} \times M_{2}$ is increased from $25 \times 25$ to $201 \times 201$. The average convergence rate is about 0.86 for $\mathrm{P}$-wave and 0.73 for $\mathrm{S}$-wave. The rate is around 0.7 for the total energy density. It is slightly slower than the previous example, since we use dimension-by-dimension for propagating discontinuities not aligned with the grids.

\begin{tabular}{ccccc}
\hline grid points $\left(M_{1} \times M_{2}\right)$ & $25 \times 25$ & $51 \times 51$ & $101 \times 101$ & $201 \times 201$ \\
\hline $\mathcal{E}^{p}$ & $1.60892 \mathrm{E}-2$ & $0.91924 \mathrm{E}-2$ & $0.53653 \mathrm{E}-2$ & $0.31354 \mathrm{E}-2$ \\
$\mathcal{E}^{s}$ & $3.65725 \mathrm{E}-2$ & $2.25543 \mathrm{E}-2$ & $1.41858 \mathrm{E}-2$ & $0.97879 \mathrm{E}-2$ \\
$E=\mathcal{E}^{p}+\mathcal{E}^{s}$ & $4.73282 \mathrm{E}-2$ & $2.98777 \mathrm{E}-2$ & $1.89317 \mathrm{E}-2$ & $1.26550 \mathrm{E}-2$ \\
\hline
\end{tabular}

Table 2: Example 6.2. $l^{1}$ error of energy density $\mathcal{E}^{p}, \mathcal{E}^{s}$ and the total energy density $E(t, \mathbf{x})$ using different meshes at $t=0.50$.

Example 6.3 The solids on two sides of the interface are the same as those in Example 6.1. But the shape of interface is like a wedge shown in Figure 10. The interface curve is given by

$$
\mathcal{F}(x, y)= \begin{cases}y-\tan \left(\frac{\pi}{2.5}\right)(x-0.18) & \text { if } y>0.07 \tan \left(\frac{\pi}{2.5}\right) \\ 0.25-x & \text { if } y<0.07 \tan \left(\frac{\pi}{2.5}\right)\end{cases}
$$

So the discontinuous wave speeds are

$$
c^{p}(x, y)=\left\{\begin{array}{lll}
\sqrt{1.2} & \text { if } \quad \mathcal{F}(x, y)>0 \\
\sqrt{2.5} & \text { if } \quad \mathcal{F}(x, y)<0
\end{array}\right.
$$

and

$$
c^{s}(x, y)=\left\{\begin{array}{lll}
\sqrt{0.5} & \text { if } & \mathcal{F}(x, y)>0 \\
1 & \text { if } & \mathcal{F}(x, y)<0
\end{array}\right.
$$

The initial conditions are given by $\mathbf{A}_{0}=(1,0.5)$ and $\nabla S_{0}=(0.20,0.25)$. So the corresponding $\mathcal{E}_{0}^{p}(\mathbf{x})$ and $\mathcal{E}_{0}^{s}(\mathbf{x})$ are

$$
\mathcal{E}_{0}^{p}(x, y)=\left\{\begin{array}{lll}
0.8587 & \text { if } & \mathcal{F}(x, y)>0 \\
0.4122 & \text { if } & \mathcal{F}(x, y)<0
\end{array}\right.
$$

and

$$
\mathcal{E}_{0}^{s}(x, y)=\left\{\begin{array}{lll}
0.4390 & \text { if } & \mathcal{F}(x, y)>0 \\
0.2195 & \text { if } \quad \mathcal{F}(x, y)<0
\end{array}\right.
$$


For exact solution, the reflection and transmission coefficients at vertical part of the interface are $\beta_{R}^{P P}=0.6392, \beta_{R}^{P S}=0.1436, \beta_{T}^{P S}=0.2171, \beta_{R}^{S S}=1$; the coefficients at slant part of the interface are $\beta_{R}^{P P}=0.7821, \beta_{R}^{P S}=0.0801, \beta_{T}^{P S}=0.1378, \beta_{R}^{S S}=1$. The graph of the exact solution at $t=0.25$ is shown in Figure 11(a).

We use the same discretization for $\boldsymbol{\delta}$-function as in the first two examples and choose the time step as $\Delta t=\frac{1}{8} \Delta x$. And the computational domain is chosen as $(x, y, \xi, \eta) \in[0,0.5] \times[0,0.5] \times[-0.5,0.3] \times[0.2,0.6]$. In this example, the interface curve is not aligned with the grid and we should treat it with the way introduced in Section 5.

The numerical energy density for $\mathrm{P}$-wave and $\mathrm{S}$-wave at $t=0.25$ is displayed in Figure 11(b), which can be compared with the corresponding exact solution. Figure 12 and Figure 14 display the sectional view along the line $y=0.2$ for energy density of P-wave and S-wave at four different times $t=0.1, t=0.18, t=0.25, t=0.3$, which show the wave behavior around the vertical part of the interface, which are similar to that in Example 6.1. Figure 13 and Figure 15 display the sectional view along the line $y=0.4$ for energy density of $\mathrm{P}$-wave and $\mathrm{S}$-wave at the same four different times, which show the wave behavior around the slant part of the interface where a more interesting pattern comes out. In both cases, the material interfaces are captured sharply.

More comparison is given in Figure 16 and Figure 17, which display the projected images into the plane for two types of waves at $t=0.1$ and $t=0.25$ respectively. Each wave pattern matches pretty well with the exact solution.

Table 3 presents the $l^{1}$ error, at $t=0.25$, of energy density for P-wave and S-wave respectively and also of total energy density $E(t, \mathbf{x})$ computed with the different meshes $M_{1} \times M_{2} \times N_{1} \times N_{2}$ where we fix $N_{1} \times N_{2}=80 \times 80$ and $M_{1} \times M_{2}$ is increased from $25 \times 25$ to $201 \times 201$. The average convergence rate is about 0.79 for $\mathrm{P}$-wave and 0.67 for S-wave. The rate is around 0.67 for the total energy density. It is slower than the first two examples, mainly due to the approximation error at the slant interface where the interface was locally moved to the nearest grid point when we define the numerical flux.

\begin{tabular}{ccccc}
\hline grid points $\left(M_{1} \times M_{2}\right)$ & $25 \times 25$ & $51 \times 51$ & $101 \times 101$ & $201 \times 201$ \\
\hline $\mathcal{E}^{p}$ & $0.72704 \mathrm{E}-2$ & $0.38293 \mathrm{E}-2$ & $0.22380 \mathrm{E}-2$ & $0.14419 \mathrm{E}-2$ \\
$\mathcal{E}^{s}$ & $0.56764 \mathrm{E}-2$ & $0.35783 \mathrm{E}-2$ & $0.22371 \mathrm{E}-2$ & $0.14595 \mathrm{E}-2$ \\
$E=\mathcal{E}^{p}+\mathcal{E}^{s}$ & $1.20187 \mathrm{E}-2$ & $0.71593 \mathrm{E}-2$ & $0.43784 \mathrm{E}-2$ & $0.28718 \mathrm{E}-2$ \\
\hline
\end{tabular}

Table 3: Example 6.3. $l^{1}$ error of energy density $\mathcal{E}^{p}, \mathcal{E}^{s}$ and the total energy density $E(t, \mathbf{x})$ using different meshes at $t=0.25$. 


\section{Conclusion}

In this paper, we extended the previous works in $[10,12]$ to the Liouville euqations for high frequency elastic waves in heterogeneous media. By building into the numerical flux the constant Hamiltonian condition and the scattering information at the interface, our scheme can effectively compute the mode conversion between $\mathrm{P}$-wave and S-wave at the interface, with a sharp numerical resolution. The numerical scheme allows a hyperbolic CFL condition, under which the scheme is positive, and stable in $l^{\infty}$ norm. Numerical experiments are carried out to study the numerical convergence and accuracy. In the future we will extend the method to three dimendional problems, where coupled systems of Liouville equations need to be solved [22].

\section{References}

[1] J.D. Achenbach, Reciprocity in Elastodynamics, Cambridge University Press 2003.

[2] K. Aki and P.G. Richards, Quantitative seismology, 2nd Edition, Univesity Science Books 2002.

[3] G. Bal, J.B. Keller, G. Papanicolaou and L. Ryzhik, Transport theory for acoustic waves with reflection and transmission at interfaces. Wave Motion, 30, 303-327, 1999.

[4] R. Burridge, Some Mathematical topics in seismology, Courant Inst. of Math. Sciences, New York, 1976.

[5] B. Engquist and Olof Runborg, Computational high frequency wave propagation, Acta Numerica, 181-266, 2003.

[6] B. Engquist, A.K. Tornberg and R. Tsai, Discretization of Dirac delta functions in level set methods, J. Comp. Phys., to appear.

[7] W.M. Ewing, W.S. Jardetzky and F. Press, Elastic waves in layered media, McGraw-Hill Book Company, INC., 1957.

[8] J.A. Hudson, The total internal reflection of SH waves. Geophys. J.R. astr. Soc., 6, 509-531, 1962.

[9] S. Jin, H.L. Liu, S. Osher and R. Tsai, Computing multi-valued physical observables for the high frequency limit of symmetric hyperbolic systems, J. Comp. Phys. 210, 497-518, 2005.

[10] S. Jin and X. Wen, Hamiltonian-preserving schemes for the Liouville equation with discontinuous potentials, Comm. Math. Sci. 3, 285-315, 2005. 
[11] S. Jin and X. Wen, Hamiltonian-preserving schemes for the Liouville equation of geometrical optics with discontinuous local wave speeds, J. Comp. Phys., to appear.

[12] S. Jin and X. Wen, Hamiltonian-preserving schemes for the Liouville equation of geometrical optics with transmissions and reflections, SIAM J. Num. Anal., submitted.

[13] S. Jin and X. Wen, Computation of Transmissions and Reflections in Geometrical Optics the Reduced Liouville Equation, Wave Motion, submitted.

[14] B.L.N. Kennett, N.J. Kerry and J.H. Woodhouse, Symmetries in the reflection and transmission of elastic waves. Geophys. J.R. astr. Soc., 52, 215-229, 1978.

[15] E.R. Lapwood and J.A. Hudson, The passage of elastic waves through an anomalous region - III. Transmission of obliquely incident body waves. Geophys. J. Roy. Astr. Soc., 40, 255-268, 1975.

[16] R.J. Leveque, Finite Volumn Methods for Hyperbolic Problems. Cambridge University Press, 2002.

[17] R.J. LeVeque and Z.L. Li, The immersed interface method for elliptic equations with discontinuous coefficients and singular sources, SIAM J. Numer. Anal. 31, 1019-1044, 1994.

[18] R.J. LeVeque and C.M. Zhang, The immersed interface method for acoustic wave equations with discontinuous coefficients, Wave Motion, Vol.25, No.3, May 1997, $237-263(27)$.

[19] L. Miller, Refraction of high frequency waves density by sharp interfaces and semiclassical measures at the boundary, J. Math. Pures Appl. IX 79, 227-269, (2000).

[20] M. Muskat and M.W. Meres, Reflection and transmission coefficients for plane waves in elastic media. geophysics, Vol.5, 1940.

[21] B. Perthame and C.W. Shu, On positivity preserving finite volume schemes for Euler equations, Numer. Math. 73: 119-130, 1996.

[22] L. Ryzhik, G. Papanicolaou and J.B. Keller, Transport equations for elastic and other waves in random media. Wave Motion, 24(4):327-370, 1996.

[23] L. Ryzhik, G. Papanicolaou and J. Keller, Transport equations for waves in a half space, Comm. PDE's, 22, 1869-1910 (1997).

[24] T. Tang and Z.H. Teng, The sharpness of Kuznetsov's $O(\sqrt{\Delta x}) L^{1}$-error estimate for monotone difference schemes. Mathematics of Computation, Vol.64, No.210, 581-589. 
[25] C.M. Zhang, The immersed interface method for elastic wave propagation in heterogeneous materials, Annual Report of the Rice Inversion Project, 1997.
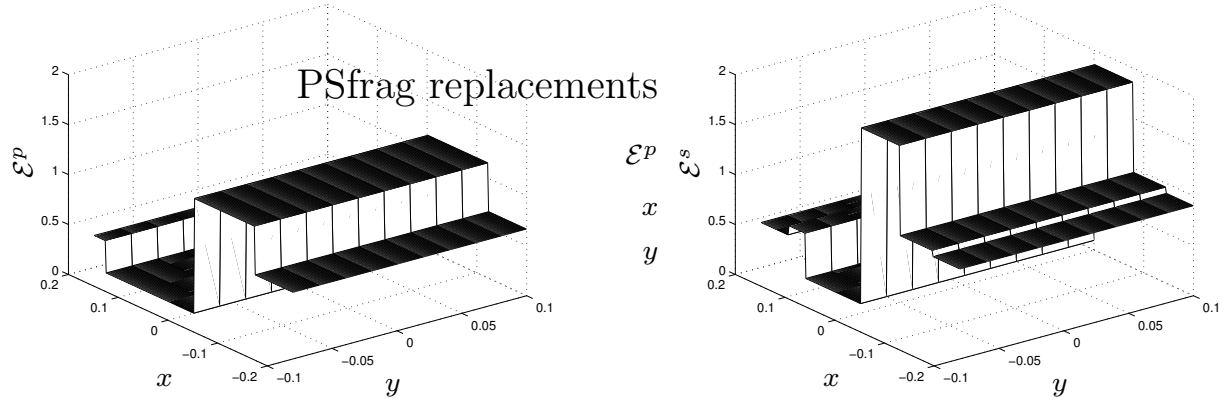

(a) Exact solution
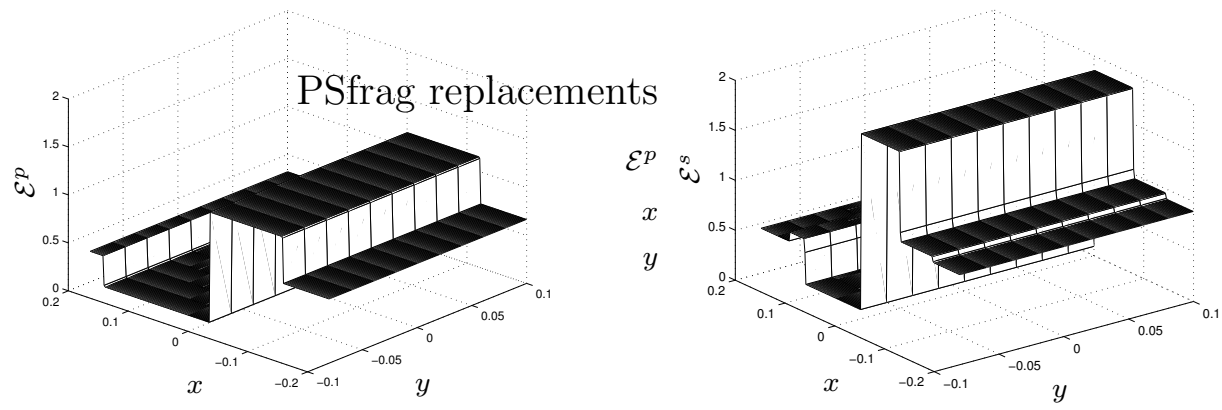

(b) Numerical solution

Figure 3: Example 6.1. Energy density of $\mathrm{P}$-wave and $\mathrm{S}$-wave at $t=0.25$. The numerical solution using the phase space mesh $801 \times 10 \times 80 \times 20$. 

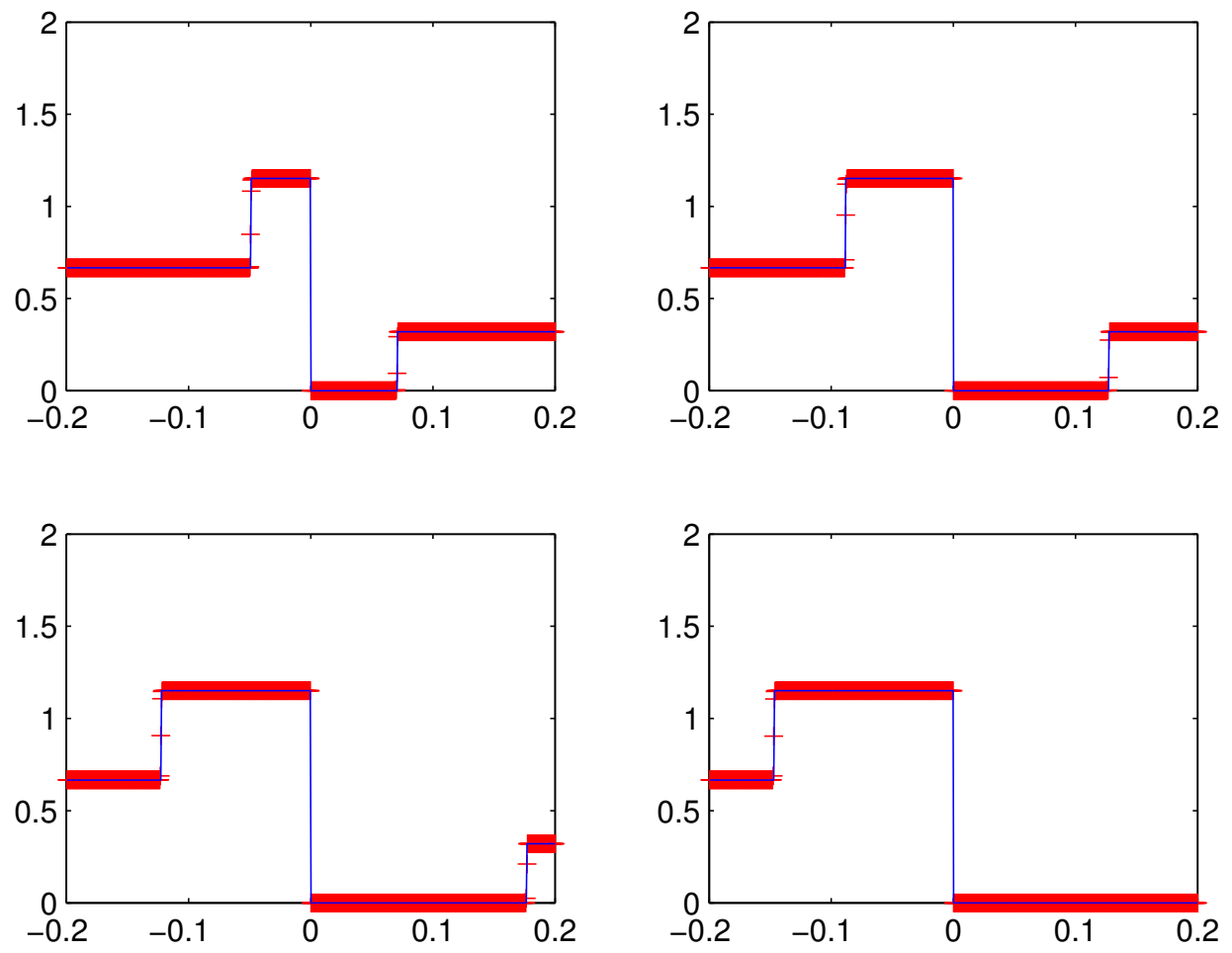

Figure 4: Example 6.1. A sectional view in $\mathrm{x}$-direction for energy density of $\mathrm{P}$-wave at four different times $t=0.1, t=0.18, t=0.25, t=0.30$ (from top left to bottom right) overlaid with the corresponding exact solution. Solid line: the exact solution; '+': the numerical solution. 

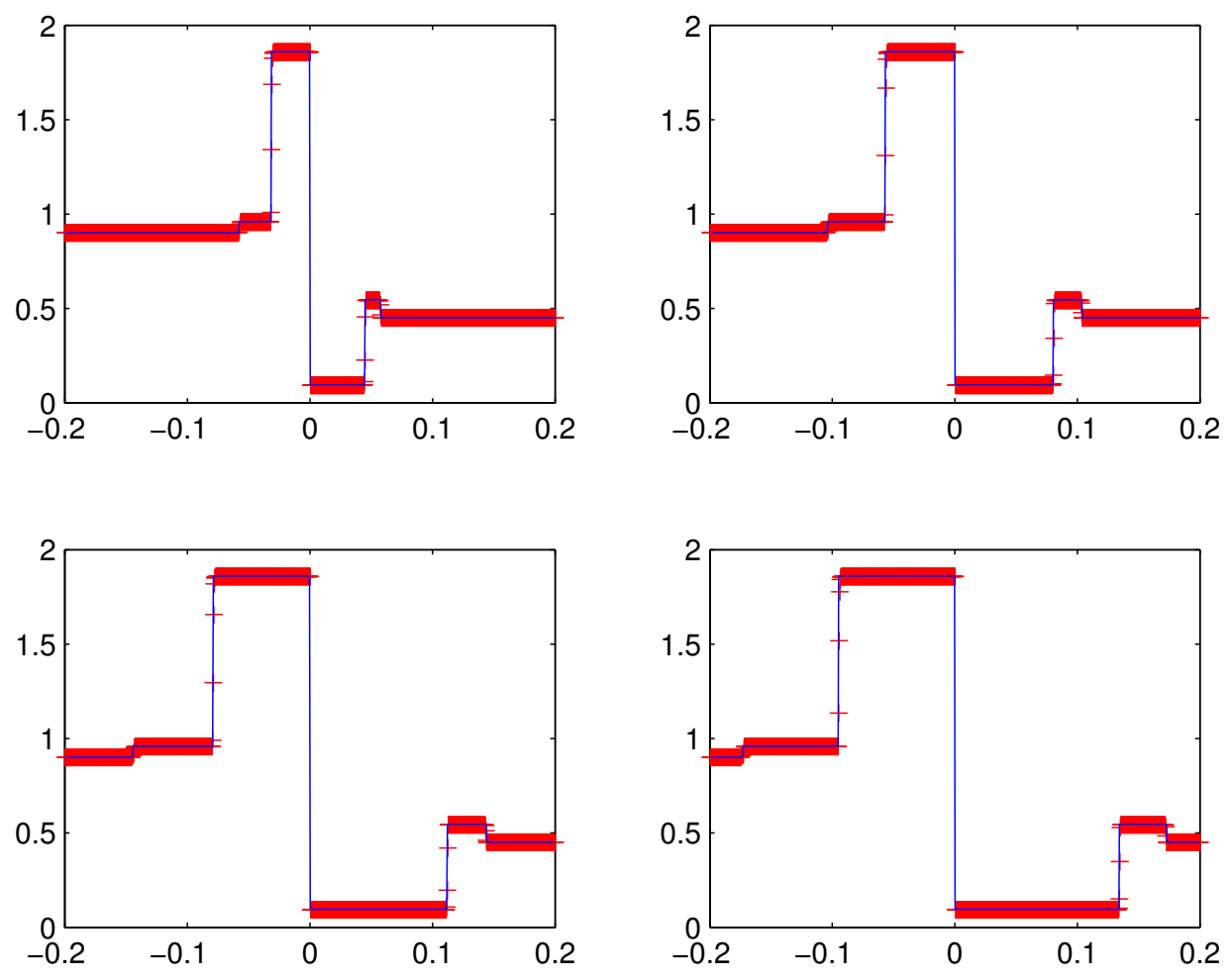

Figure 5: Example 6.1. A sectional view in x-direction for energy density of S-wave at four different times $t=0.1, t=0.18, t=0.25, t=0.30$ (from top left to bottom right) overlaid with the corresponding exact solution. Solid line: the exact solution; '+': the numerical solution. 

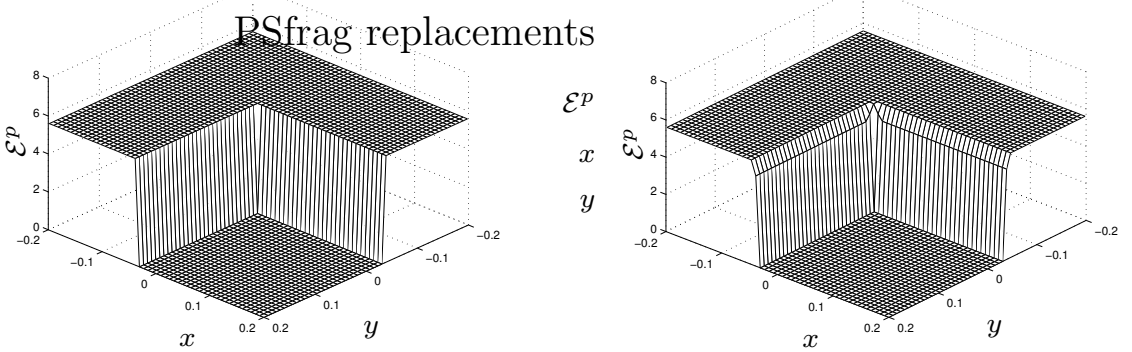

(a) $\mathrm{t}=0.10$
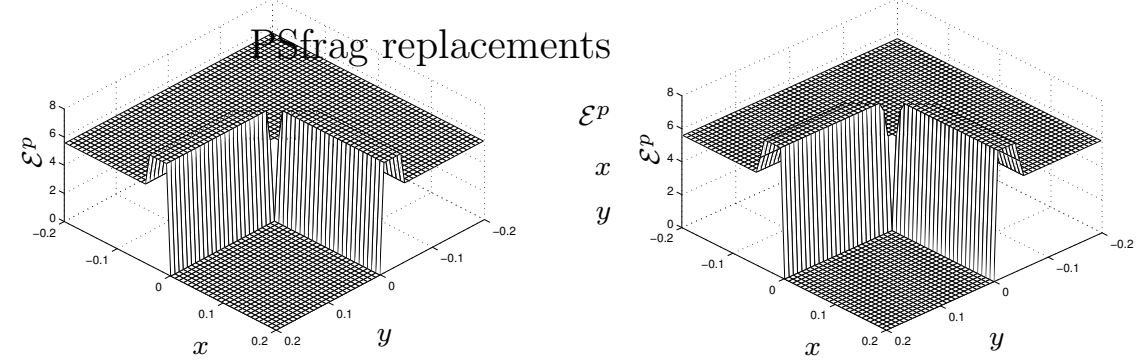

(b) $\mathrm{t}=0.23$
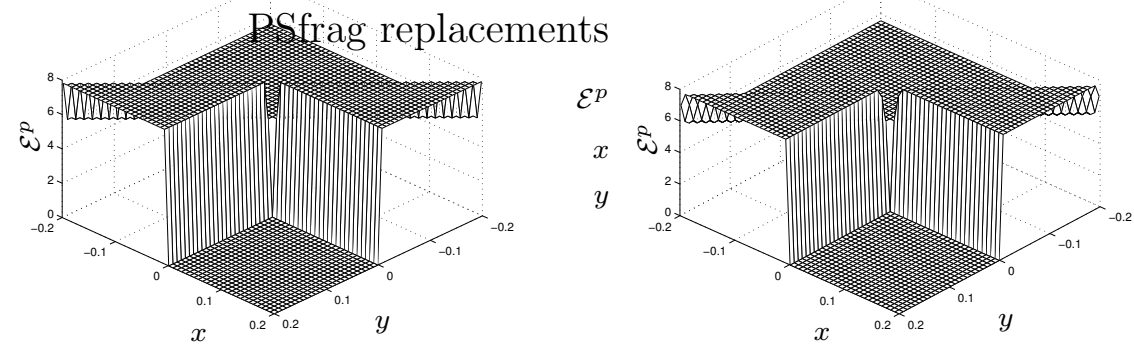

(c) $\mathrm{t}=0.50$

Figure 6: Example 6.2. Energy density of P-wave at different times. Left: Exact solution; Right: Numerical solution using the phase mesh $201 \times 201 \times 80 \times 80$. 

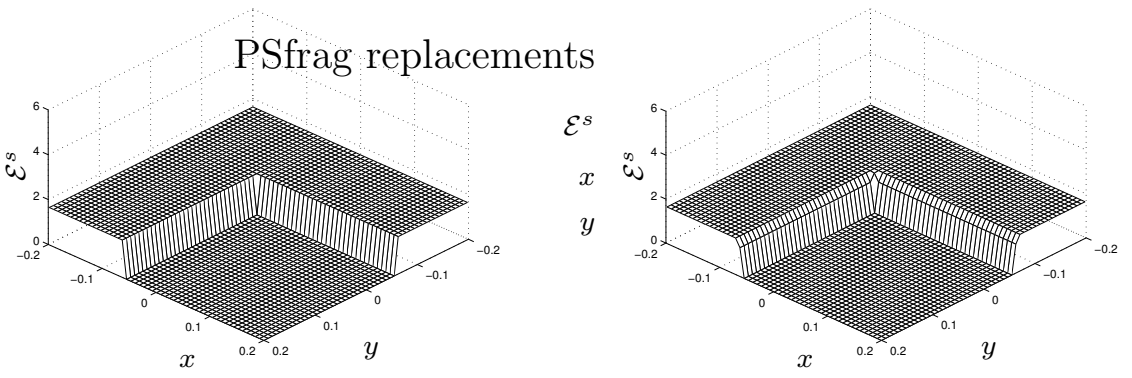

(a) $\mathrm{t}=0.10$
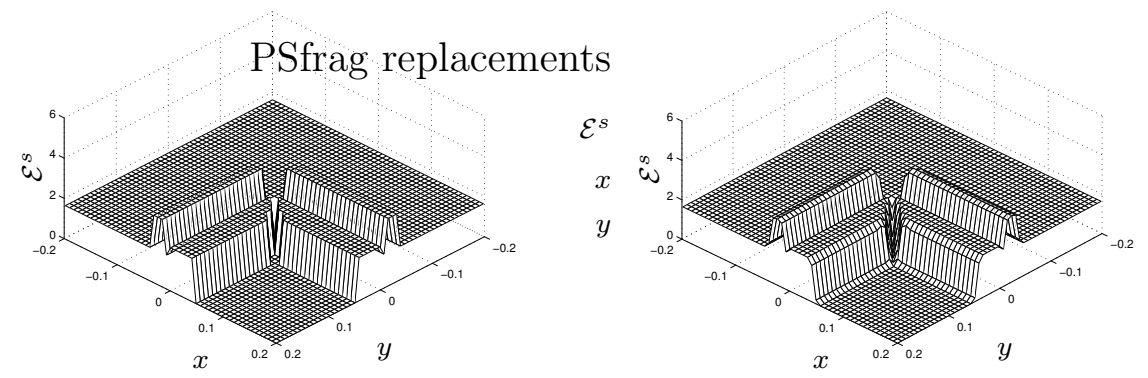

(b) $\mathrm{t}=0.23$
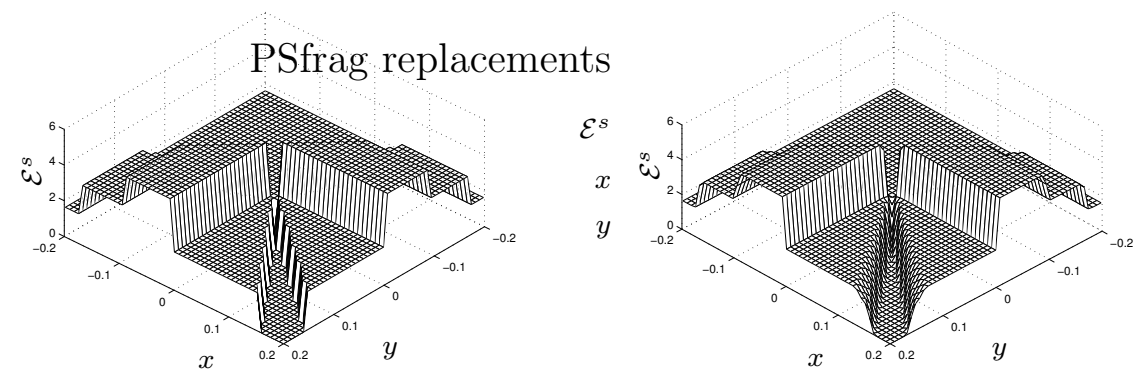

(c) $\mathrm{t}=0.50$

Figure 7: Example 6.2. Energy density of S-wave at different times. Left: Exact solution; Right: Numerical solution using the phase mesh $201 \times 201 \times 80 \times 80$. 

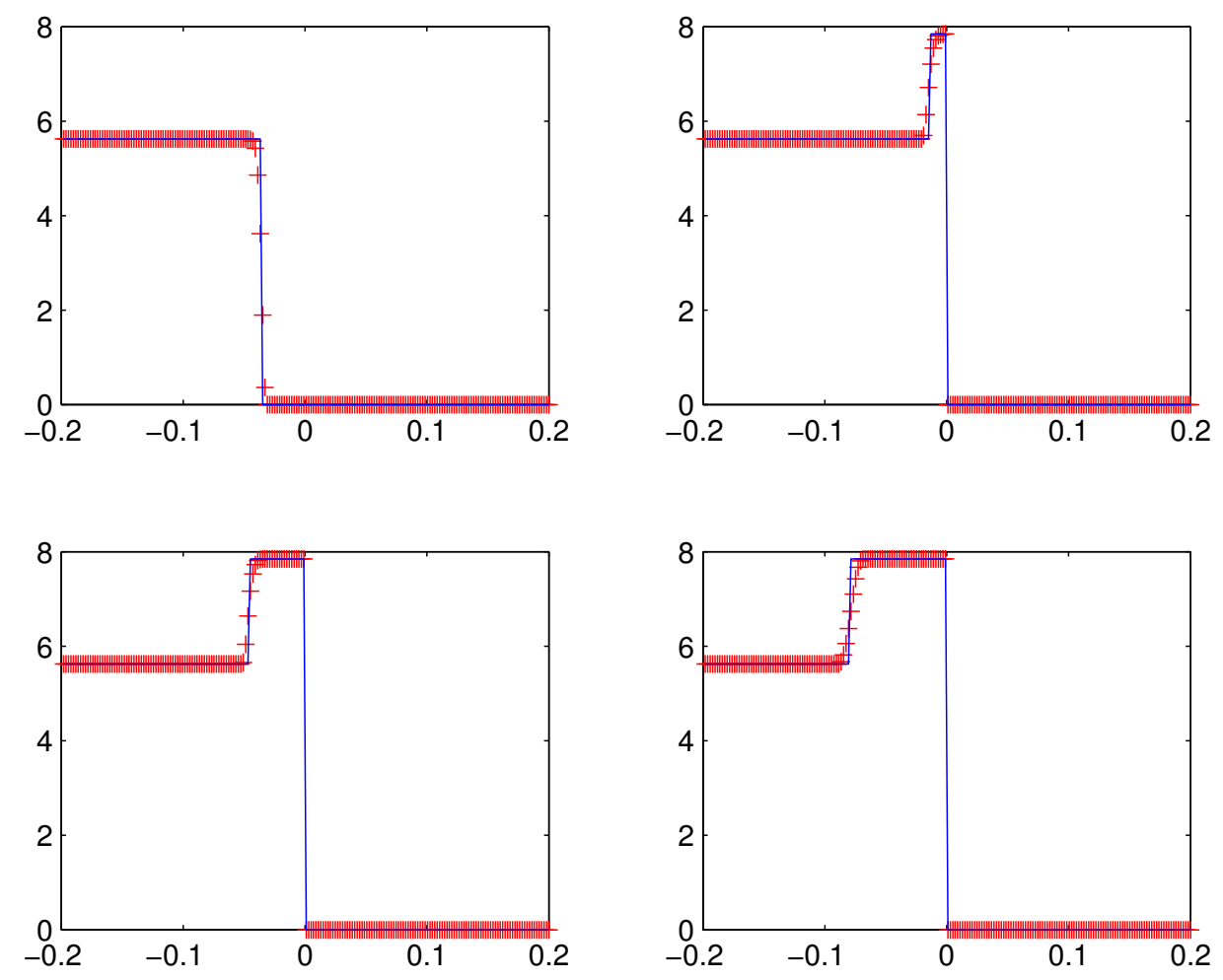

Figure 8: Example 6.2. A sectional view along the line $y=0.08$ for energy density of Pwave at four different times $t=0.1, t=0.18, t=0.23, t=0.45$ (from top left to bottom right) overlaid with the corresponding exact solution. Solid line: the exact solution; '+': the numerical solution. 

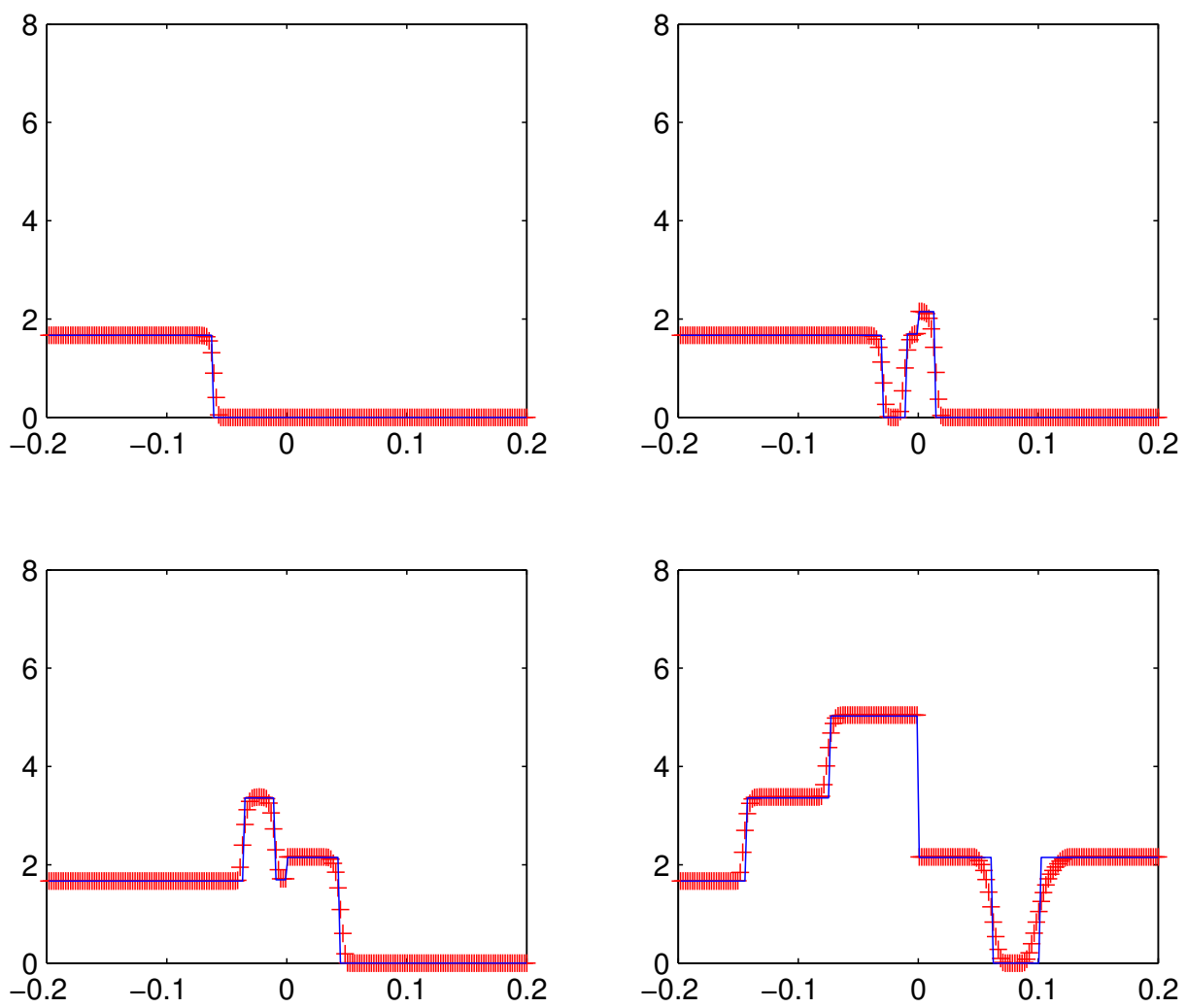

Figure 9: Example 6.2. A sectional view along the line $y=0.08$ for energy density of Swave at four different times $t=0.1, t=0.18, t=0.23, t=0.45$ (from top left to bottom right) overlaid with the corresponding exact solution. Solid line: the exact solution; '+': the numerical solution. 


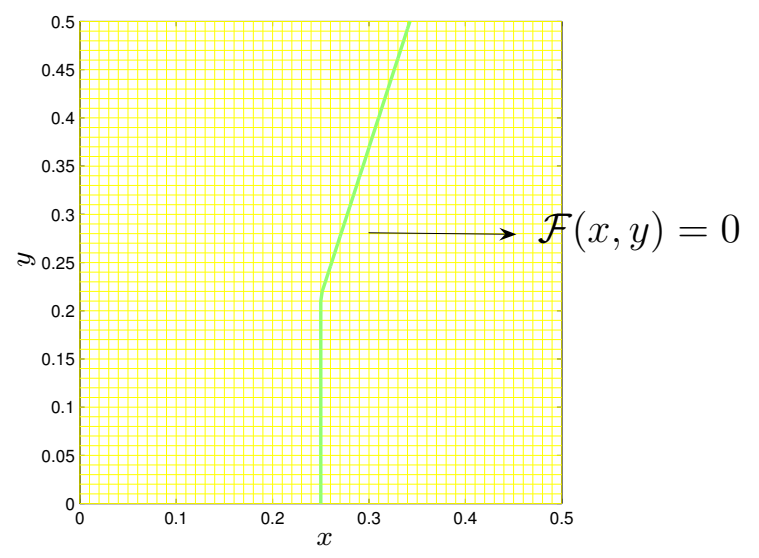

Figure 10: The interface curve of Example 6.3 in its computation domain.
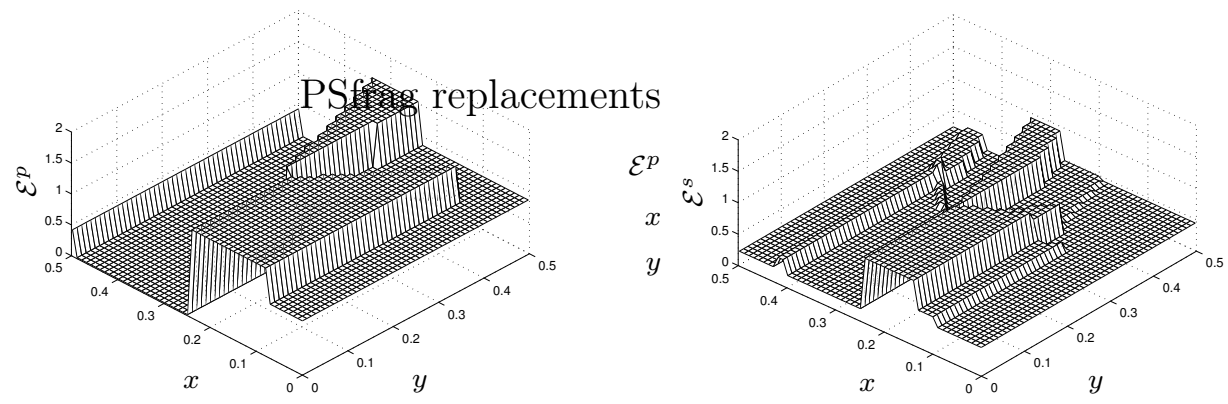

(a) Exact solution
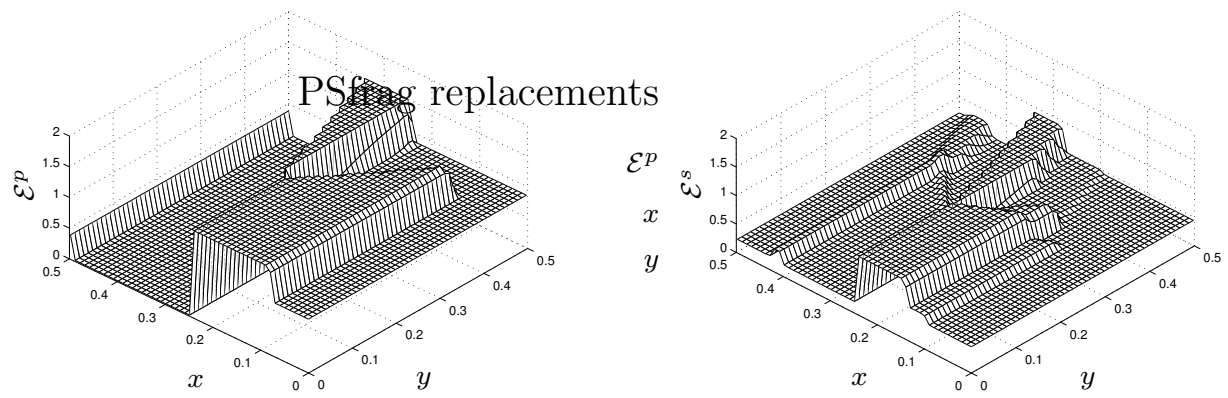

(b) Numerical solution

Figure 11: Example 6.3. Energy density of $\mathrm{P}$-wave and $\mathrm{S}$-wave at $t=0.25$. The numerical solution using the phase space mesh $201 \times 201 \times 80 \times 80$. 

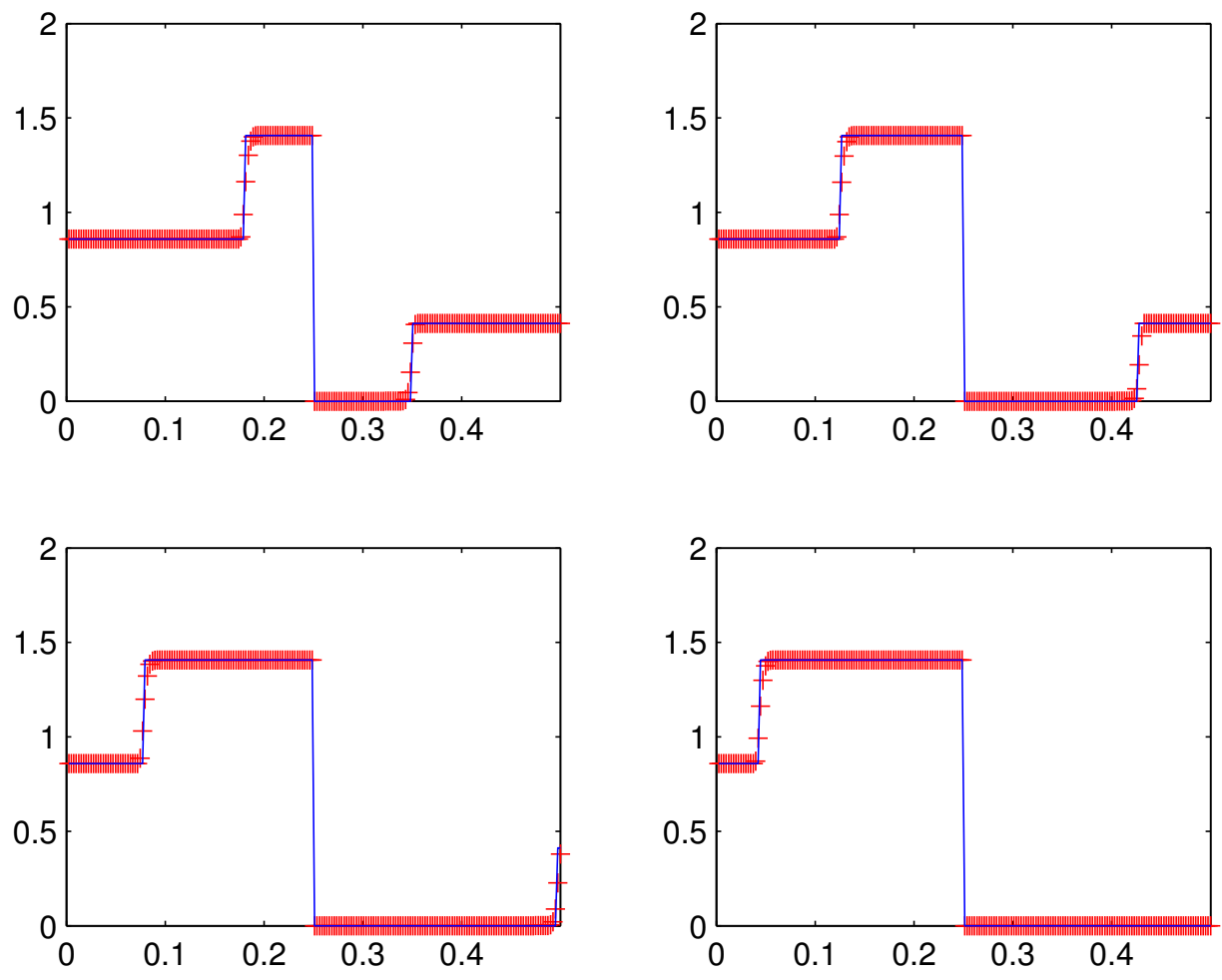

Figure 12: Example 6.3. A sectional view along $y=0.2$ for energy density of $\mathrm{P}$-wave at four different times $t=0.1, t=0.18, t=0.25, t=0.30$ (from top left to bottom right) overlaid with the corresponding exact solution. Solid line: the exact solution; ' + ': the numerical solution. 

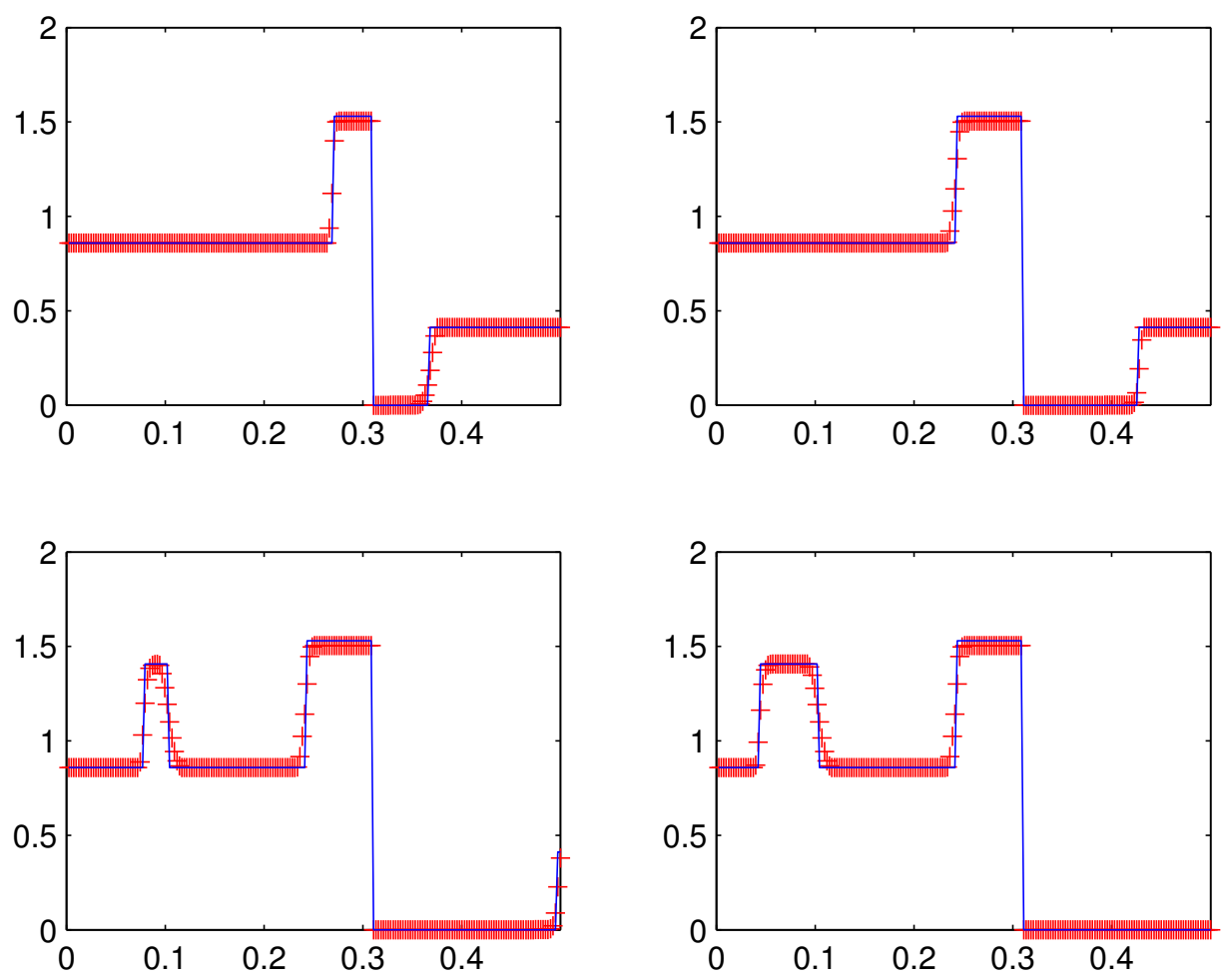

Figure 13: Example 6.3. A sectional view along $y=0.4$ for energy density of $\mathrm{P}$-wave at four different times $t=0.1, t=0.18, t=0.25, t=0.30$ (from top left to bottom right) overlaid with the corresponding exact solution. Solid line: the exact solution; ' + ': the numerical solution. 

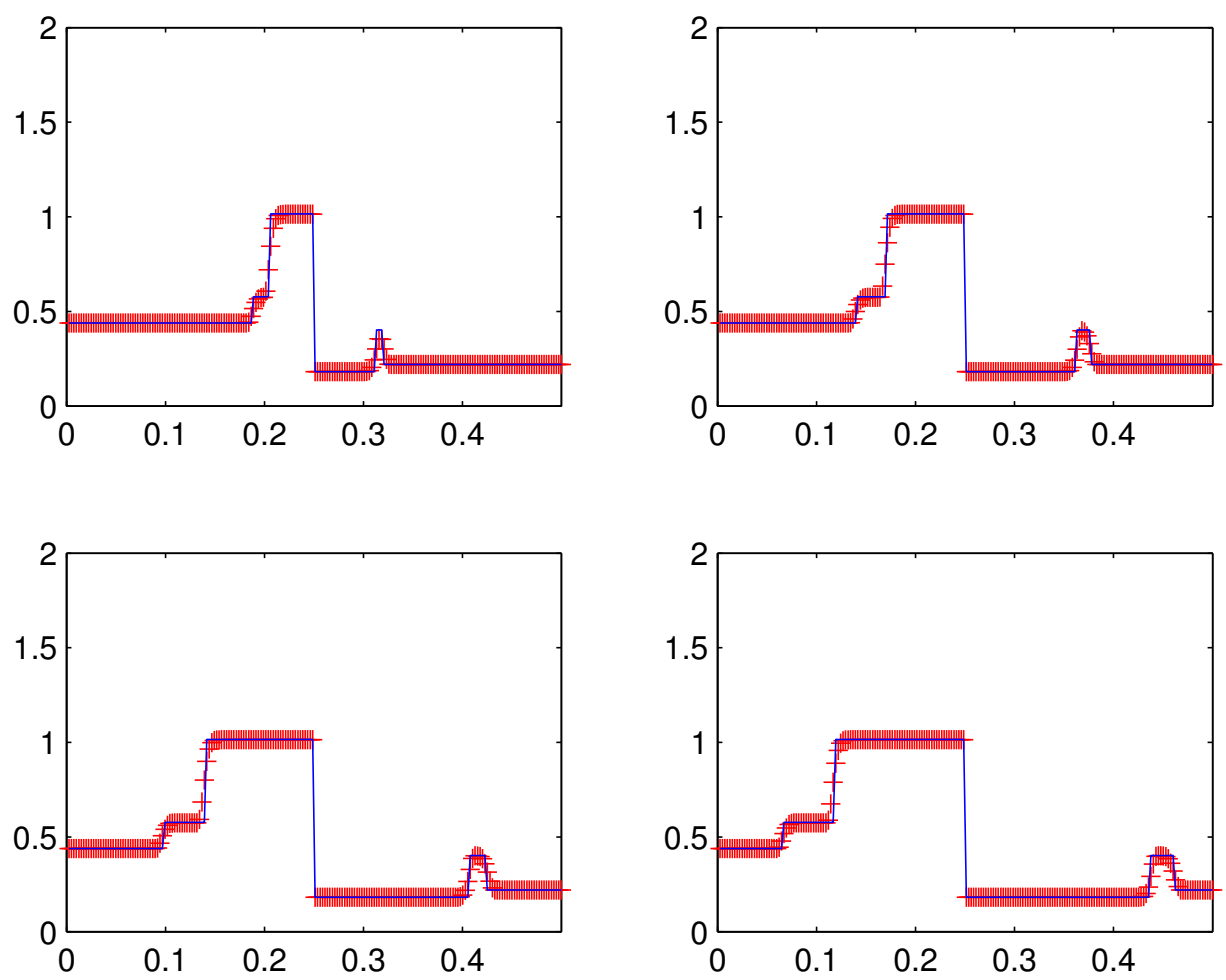

Figure 14: Example 6.3. A sectional view along $y=0.2$ for energy density of S-wave at four different times $t=0.1, t=0.18, t=0.25, t=0.30$ (from top left to bottom right) overlaid with the corresponding exact solution. Solid line: the exact solution; ' + ': the numerical solution. 

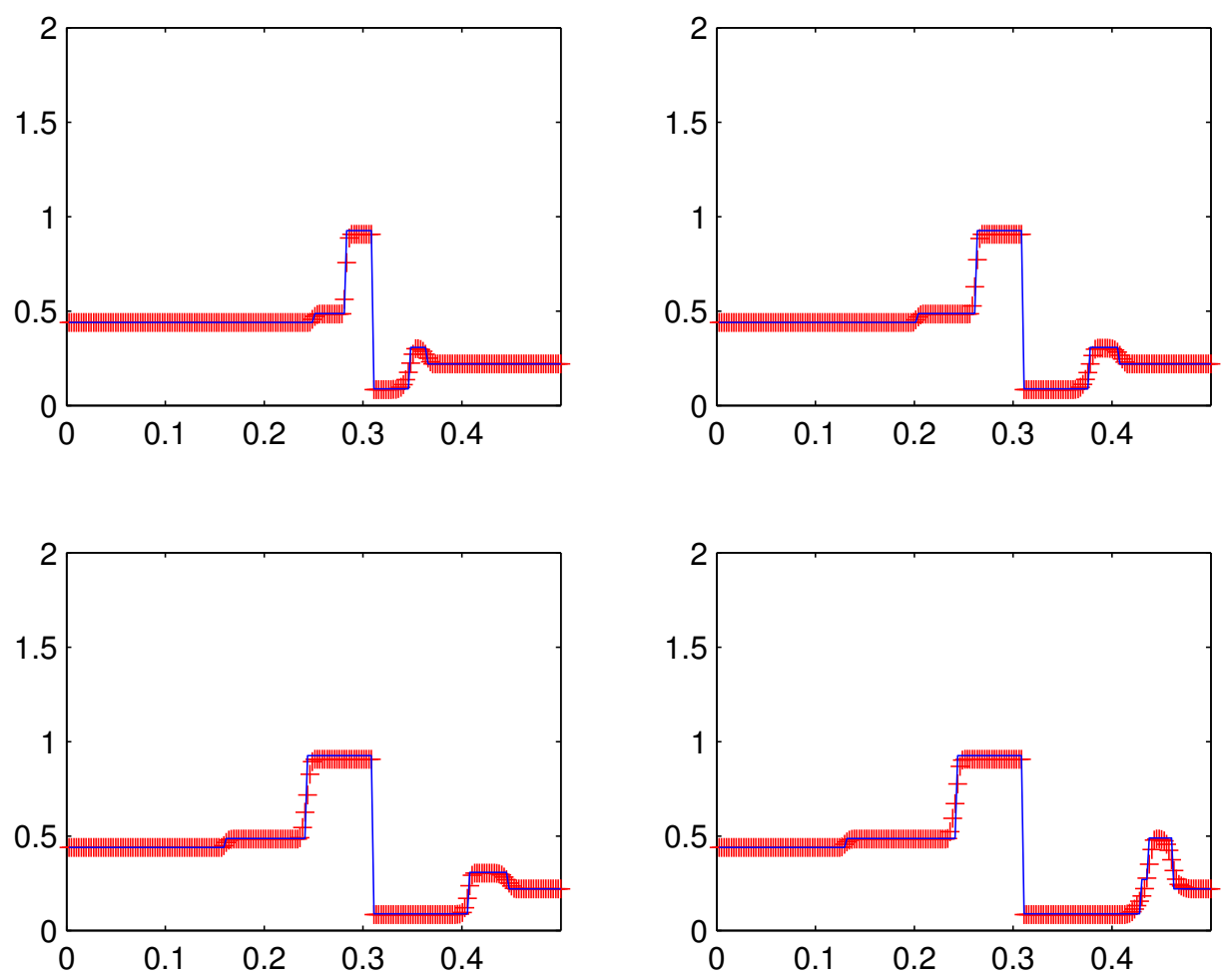

Figure 15: Example 6.3. A sectional view along $y=0.4$ for energy density of S-wave at four different times $t=0.1, t=0.18, t=0.25, t=0.30$ (from top left to bottom right) overlaid with the corresponding exact solution. Solid line: the exact solution; ' + ': the numerical solution. 



(a) $\mathrm{t}=0.1$
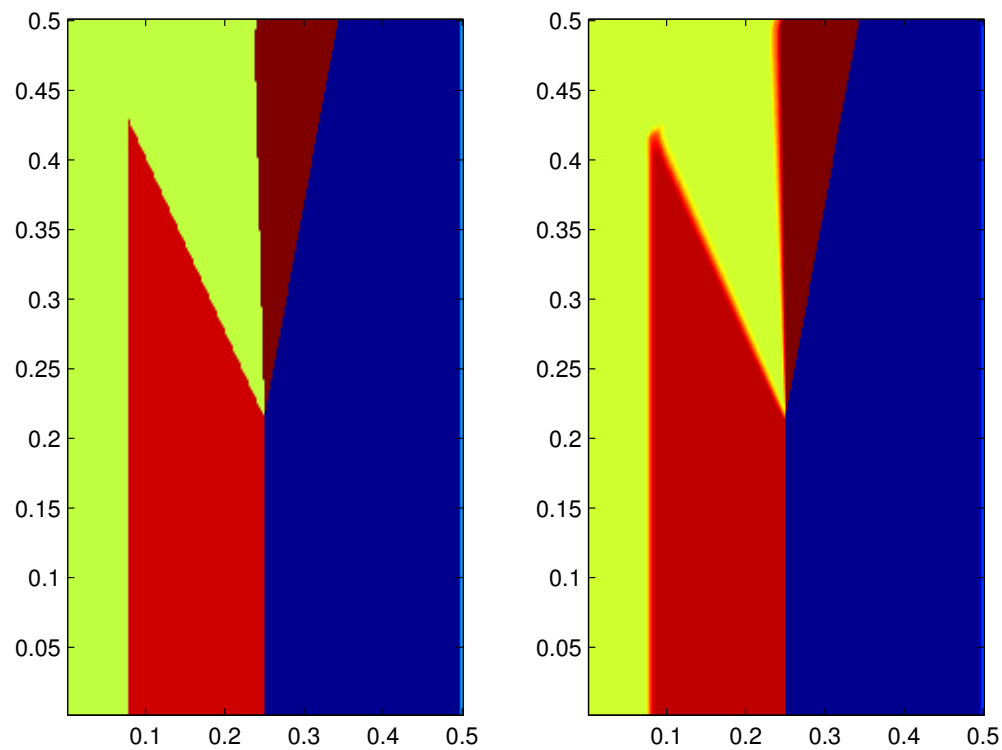

(b) $\mathrm{t}=0.25$

Figure 16: Example 6.3. The scaled P-wave image at $t=0.1$ and $t=0.25$. Left: Exact solution; Right: Numerical solution. 



(a) $\mathrm{t}=0.1$
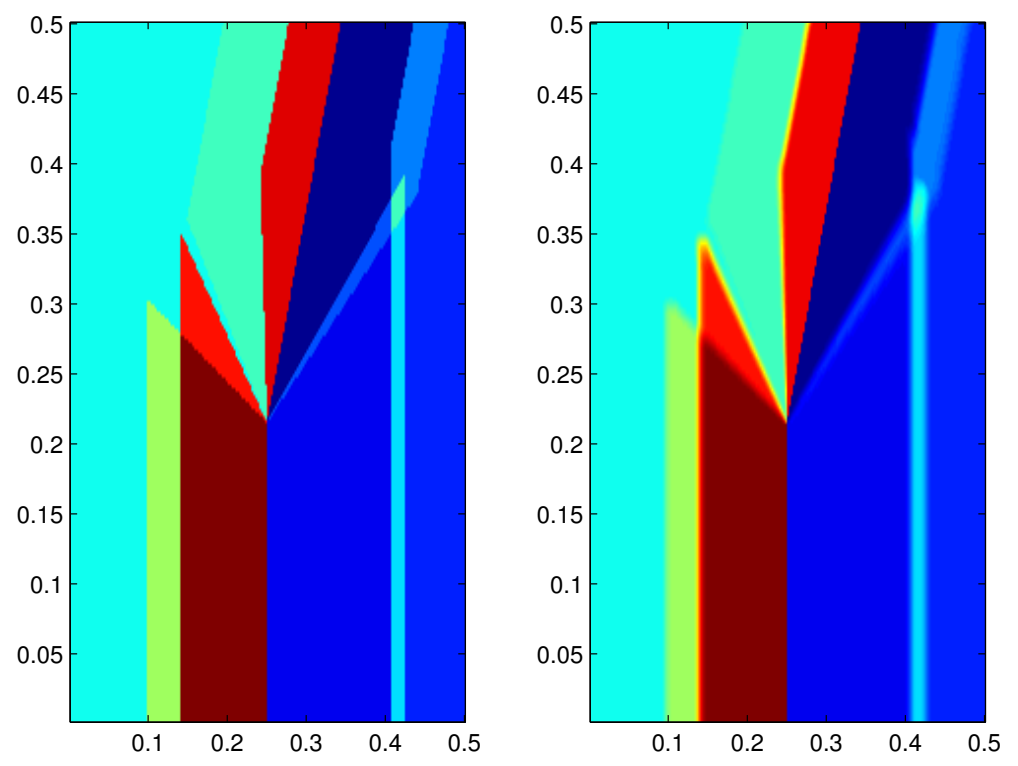

(b) $\mathrm{t}=0.25$

Figure 17: Example 6.3. The scaled S-wave image at $t=0.1$ and $t=0.25$. Left: Exact solution; Right: Numerical solution. 